\title{
Research Paper The evaluation of immunotherapy and chemotherapy treatment on melanoma: a network meta-analysis
}

\author{
BaSang CiRen ${ }^{1}$, Xinhua Wang ${ }^{2}$, Ziwen Long ${ }^{3}$ \\ ${ }^{1}$ Department of Medicine, Shigatse People's Hospital, Shigatse, Tibet, 85700, China \\ ${ }^{2}$ Department of Dermatology, Shigatse People's Hospital, Shigatse, Tibet, 85700, China \\ ${ }^{3}$ Department of Gastric Cancer and Soft-Tissue Sarcoma Sugery, Fudan University Shanghai Cancer Center; Department of \\ Oncology, Shanghai Medical College, Fudan University, Shanghai, 200032, China
}

Correspondence to: Ziwen Long, email: liuyi_jinan@163.com

Keywords: melanoma, chemotherapy, immunotherapy, network meta-analysis

Received: August 10, 2016

Accepted: October 17, 2016

Published: November 10, 2016

\section{ABSTRACT}

Background: Melanoma is a highly malignant tumor that develops from a neural crest derivative called melanocytes. Chemotherapy is recommended for patients with stage III/IV melanoma. Immunomodulation has also been shown to effectively improve the survival rate of such patients. In the current study, we aimed to perform a network meta-analysis on the therapeutic value of chemotherapy and immunotherapy on melanoma.

Results: Twenty randomized controlled trials (RCTs) were enrolled in the study. Our Results indicated that ipilimumab + nivolumab had the highest response rate among all therapies, pembrolizumab also had a good efficacy with an excellent tolerance. Chemotherapy had a low response rate, high adverse effects and progressive diseases qualities, therefore it is not recommended as a preferred treatment for patients with advanced melanoma.

Methods: The Cochrane library, PubMed and Embase databases were searched for relevant articles. Results of the pair-wise meta-analysis were illustrated by odd ratios (ORs) and corresponding 95\% confidence intervals (CIs). Network meta-analysis was performed using a random-effects model under Bayesian framework. Results were illustrated by cumulative ORs and corresponding $95 \%$ credible interval (CrIs). The probabilities and outcomes of each treatment were ranked and summarized using the surface under the cumulative ranking curve (SUCRA).

Conclusions: We recommend pembrolizumab as the preferred treatment due to its high efficacy and low adverse effects, combination of ipilimumab and nivolumab could be used in severe symptoms.

\section{INTRODUCTION}

Melanoma develops from melanocytes typically in the skin, but has also been reported to occur on mucosal surfaces where neural crest cells migrate, such as the mouth, pleura and iris $[1,2]$. Melanoma is the most common cancer in young adults aged 25 to 29 [3], with an age-standardized incidence rate of $10.2 \%$ for males and $9.8 \%$ for females in developed countries [4], and 73,780 new cases of melanoma and 9,940 melanoma-related deaths in the United States [5].

Both environmental and genetic conditions are considered as risk factors. Family history, skin type, density of freckles, skin color, eye and hair color, pre-malignant and skin cancer lesions, and actinic damage indicators are all significantly related to melanoma susceptibility [6]. Gene mutations combined with environmental factors, particularly the exposure to UV light, may contribute to the onset of melanoma. Oncogenes including BRAF and the microphthalmia-associated transcription factor (MITF) pathway play an important role in the pathogenesis of melanoma [6].

Surgery is recommended for patients with dissectible lesions and oligometastatic melanoma, whereas, chemotherapy and radiotherapy are recommended for patients with non-dissectible melanoma, and particularly 
for those already in stage III or IV [7]. Besides these treatments, immunomodulation has also been observed to be effective in improving the survival rate of patients with stage IV melanoma [8].

Sentinel node biopsy is the current procedure used by the American Joint Committee on Cancer (AJCC) and Union for International Cancer Control (UICC) to treat and categorize patients with melanoma [9]. Results of the sentinel node biopsy are a strong prognostic factor in melanoma treatment [10]. Tumor thickness in millimeters (Breslow's depth), depth related skin structures (Clark level), ulceration, lymphatic/perineural invasion, tumorinfiltrating lymphocytes, location of lesion, satellite lesions, regional or distant metastasis, and the type of melanoma are all factors that may influence the prognosis of melanoma [7]. The age-standardized mortality rate of melanoma is about $2 \%$ [4]. However, for advanced melanoma, the fiveyear survival rate could be as low as $10 \%$ [8].

Several trials concerning the effect of immunomodulation and chemotherapy on melanoma have been performed. The therapeutic value of chemotherapy, cytotoxic T-lymphocyte associated protein 4 (CTLA4) and programmed death1 (PD-1) antibodies has also been assessed in various other studies [8, 11, 12]. However, we failed to find a robust study that compares their effect on melanoma and thus clinical practice on treatment selection is required for patients with advanced melanoma. In current study, we aimed to perform a network metaanalysis concerning the therapeutic value of chemotherapy and immunotherapy on melanoma.

\section{RESULTS}

\section{Study characteristics}

As shown in Table 1 and Supplementary Figure S1, in the present meta-analysis we conducted 20 RCTs concerning the effect of immunotherapy and chemotherapy on patients with melanoma [13-32]. A total of 6,442 cases were involved and interventions were categorized into chemotherapy, ipilimumab $3 \mathrm{mg} / \mathrm{kg}$, ipilimumab $10 \mathrm{mg} / \mathrm{kg}$, tremelimumab $10 \mathrm{mg} / \mathrm{kg}$, tremelimumab 15 $\mathrm{mg} / \mathrm{kg}$, nivolumab $3 \mathrm{mg} / \mathrm{kg}$, pembrolizumab $10 \mathrm{mg} /$ $\mathrm{kg}$, pembrolizumab $2 \mathrm{mg} / \mathrm{kg}$, ipilimumab+nivolumab and ipilimumab+chemotherapy. An evidence network of eligible comparisons regarding outcomes mentioned above all was plotted in Figure 1 and Figure 2.

\section{Clinical outcomes}

In traditional pair-wise meta-analysis (Table 2), we observed that chemotherapy had a lower CR and PR rate than nivolumab $3 \mathrm{mg} / \mathrm{kg}$, pembrolizumab $10 \mathrm{mg} / \mathrm{kg}$ and pembrolizumab $2 \mathrm{mg} / \mathrm{kg}$. CR, PR rate and ORR were also higher in patients under nivolumab $3 \mathrm{mg} / \mathrm{kg}$ treatment than chemotherapy $(\mathrm{OR}=6.51,95 \% \mathrm{CI}: 1.95-21.76$;
$\mathrm{OR}=2.57,95 \% \mathrm{CI}=1.76-3.75 ; \mathrm{OR}=2.92,95 \% \mathrm{CI}:$ $2.02-4.21)$ and ipilimumab $3 \mathrm{mg} / \mathrm{kg}(\mathrm{OR}=3.99,95 \% \mathrm{CI}$ $=1.72$ to $9.26 ; \mathrm{OR}=2.07,95 \% \mathrm{CI}=1.449$ to $2.97 ; \mathrm{OR}=$ $2.29,95 \% \mathrm{CI}=1.63$ to 3.22 ). They were also higher for ipilimumab+nivolumab compared to ipilimumab $3 \mathrm{mg} /$ $\mathrm{kg}$. The disease was also found to be more stable under ipilimumab treatment than in nivolumab $3 \mathrm{mg} / \mathrm{kg}$ and ipilimumab+nivolumab treatment $(\mathrm{OR}=0.49,95 \% \mathrm{CI}$ : $0.32-0.76$; OR $=0.55,95 \%$ CI: 0.37-0.80). Besides, the disease was more progressive under Ipilimumab $3 \mathrm{mg} / \mathrm{kg}$ $(\mathrm{OR}=0.45,95 \% \mathrm{CI}=0.33$ to 0.60$)$ or Nivolumab $3 \mathrm{mg} /$ $\mathrm{kg}(\mathrm{OR}=0.60,95 \% \mathrm{CI}=0.43$ to 0.84$)$ compared with that under Ipilimumab+Nivolumab. When comparing ORR, the application of ipilimumab+nivolumab was also observed to be more effective than the single use of nivolumab $(\mathrm{OR}=1.32,95 \% \mathrm{CI}$ : $1.01-1.73)$. In regards to $\mathrm{CR}$ rate, tremelimumab $15 \mathrm{mg} / \mathrm{kg}$ was significantly more effective than tremelimumab $10 \mathrm{mg} / \mathrm{kg}(\mathrm{OR}=2.48$, 95\% CI: 1.64-3.76). However, other outcomes were not significantly approved.

Our network meta-analysis combined both direct and indirect evidence into a single comparison to facilitate comparisons between interventions without a RCT. We observed that chemotherapy was less effective than nivolumab $3 \mathrm{mg} / \mathrm{kg}$, pembrolizuma $10 \mathrm{mg} / \mathrm{kg}$, pembrolizuma $2 \mathrm{mg} / \mathrm{kg}$, ipilimumab+nivolumab as well as ipilimumab + chemotherapy when comparing both CR and PR (Table 3). And $3 \mathrm{mg} / \mathrm{kg}, 10 \mathrm{mg} / \mathrm{kg}$ ipilimumab and ipilimumab + chemotherapy were found to have a lower PR than nivolumab $3 \mathrm{mg} / \mathrm{kg}$, pembrolizuma $10 \mathrm{mg} / \mathrm{kg}$, pembrolizuma $2 \mathrm{mg} / \mathrm{kg}$ and ipilimumab + nivolumab. Similar results were observed in patients under tremelimumab $15 \mathrm{mg} / \mathrm{kg}$ treatment, which has a lower $\mathrm{CR}$ and PR rate than the four therapies mentioned above. The single application of either ipilimumab or nivolumab had a lower CR than when combined (ipilimumab+ nivolumab). This result is consistent with that of pair-wise meta-analysis. Results from the network meta-analysis concerning clinical outcomes were illustrated in Figure 3 and Figure 4.

\section{Adverse effects}

We deemed fatigue, pruritus, rash, diarrhea and nausea as five important conditions in measuring adverse effects. Using traditional meta-analysis we observed the following (Table 4): Patients under chemotherapy were more likely to demonstrate symptoms of adverse effects than patients under immunotherapy. The occurrence of fatigue, diarrhea and rash was higher in chemotherapy patients than ipilimumab $3 \mathrm{mg} / \mathrm{kg}$ patients (fatigue: OR $=1.32$, 95\% CI: 1.03- 1.70; diarrhea: OR $=1.63,95 \%$ CI: 1.27-2.08; rash: $\mathrm{OR}=2.32,95 \% \mathrm{CI}: 1.73-3.10)$. Ipilimumab $3 \mathrm{mg} / \mathrm{kg}$ has a lower possibility of pruritus and diarrhea than nivolumab $3 \mathrm{mg} / \mathrm{kg}$ and pembrolizumab $10 \mathrm{mg} / \mathrm{kg}$ (pruritus: $\mathrm{OR}=0.53,95 \% \mathrm{CI}: 0.38-0.76, \mathrm{OR}=$ 
Table 1: Summary of study design characteristics

\begin{tabular}{|c|c|c|c|c|c|}
\hline Study & Trial ID & Trial Phase & Case & Intervention & Outcome \\
\hline Weber, 2015 & NCT01721746 & III & 405 & $\begin{array}{l}\text { Nibolumab } 3 \mathrm{mg} / \mathrm{kg} \text { vs. } \\
\text { Chemotherapy }\end{array}$ & $\begin{array}{l}\text { CR; PR; SD; PD; ORR; AAE; } \\
\text { Fatigue; Pruritus; Diarrhea; } \\
\text { Nausea }\end{array}$ \\
\hline Robert, 2015 & NCT01866319 & III & 834 & $\begin{array}{l}\text { Pembrolizumab } 10 \mathrm{mg} / \mathrm{kg} \text { vs. } \\
\text { Ipilimumab } 3 \mathrm{mg} / \mathrm{kg}\end{array}$ & Fatigue; Pruritus; Rash; Diarrhea \\
\hline Robert, 2015 & NCT01721772 & III & 418 & $\begin{array}{l}\text { Nivolumab } 10 \mathrm{mg} / \mathrm{kg} \text { vs. } \\
\text { Chemotherapy }\end{array}$ & $\begin{array}{l}\mathrm{CR} \text {; PR; SD; PD; ORR; AAE; } \\
\text { Fatigue; Pruritus; Rash; Diarrhea; } \\
\text { Nausea }\end{array}$ \\
\hline Ribas, 2015 & NCT01704287 & II & 540 & $\begin{array}{l}\text { Pembrolizumab } 2 \mathrm{mg} / \mathrm{kg} \text { vs. } \\
\text { Pembrolizumab } 10 \mathrm{mg} / \mathrm{kg} \text { vs. } \\
\text { Chemotherapy }\end{array}$ & $\begin{array}{l}\text { CR; PR; SD; PD; AAE; Fatigue; } \\
\text { Pruritus; Rash; Diarrhea; Nausea }\end{array}$ \\
\hline Postow, 2015 & NCT01927419 & III/IV & 109 & $\begin{array}{l}\text { Ipilimumab } 3 \mathrm{mg} / \mathrm{kg}+\mathrm{Nivolumab} \\
1 \mathrm{mg} / \mathrm{kg} \text { vs. Ipilimumab } 3 \mathrm{mg} / \mathrm{kg}\end{array}$ & $\begin{array}{l}\text { CR; PR; SD; PD; ORR; AAE; } \\
\text { Fatigue; Pruritus; Rash; Diarrhea; } \\
\text { Nausea }\end{array}$ \\
\hline Larkin, 2015 & NCT01844505 & III & 945 & $\begin{array}{l}\text { Nivolumab } 3 \mathrm{mg} / \mathrm{kg} \text { vs. } \\
\text { Ipilimumab } 3 \mathrm{mg} / \mathrm{kg}+\text { Nivolumab } \\
1 \mathrm{mg} / \mathrm{kg} \text { vs. Ipilimumab } 3 \mathrm{mg} / \mathrm{kg}\end{array}$ & $\begin{array}{l}\text { CR; PR; SD; PD; ORR; AAE; } \\
\text { Fatigue; Pruritus; Rash; Diarrhea; } \\
\text { Nausea }\end{array}$ \\
\hline Eggmont, 2015 & NCT00636168 & III & 951 & $\begin{array}{l}\text { Ipilimumab } 10 \text { mg/kg vs. } \\
\text { Chemotherapy }\end{array}$ & $\begin{array}{l}\text { AAE; Fatigue; Pruritus; Rash; } \\
\text { Diarrhea; Nausea }\end{array}$ \\
\hline Topalian, 2014 & NCT00730639 & - & 37 & $\begin{array}{l}\text { Nivolumab } 3 \mathrm{mg} / \mathrm{kg} \text { vs. } \\
\text { Nivolumab } 10 \mathrm{mg} / \mathrm{kg}\end{array}$ & $\begin{array}{l}\text { SD; ORR; AAE; Fatigue; } \\
\text { Pruritus; Rash; Diarrhea; Nausea }\end{array}$ \\
\hline Robert, 2014 & NCT01295827 & I & 173 & $\begin{array}{l}\text { Pembrolizumab } 2 \mathrm{mg} / \mathrm{kg} \text { vs. } \\
\text { Pembrolizumab } 10 \mathrm{mg} / \mathrm{kg}\end{array}$ & $\begin{array}{l}\text { CR; PR; SD; PD; ORR; Fatigue; } \\
\text { Rash; Diarrhea; }\end{array}$ \\
\hline Hodi, 2014 & NCT01134614 & III/IV & 245 & $\begin{array}{l}\text { Ipilimumab } 10 \mathrm{mg} / \\
\mathrm{kg}+\text { Chemotherapy vs. } \\
\text { Ipilimumab } 10 \mathrm{mg} / \mathrm{kg}\end{array}$ & $\begin{array}{l}\text { CR; PR; SD; PD; ORR; Fatigue; } \\
\text { Pruritus; Rash; Diarrhea; Nausea }\end{array}$ \\
\hline Ribas, 2013 & NCT00257205 & III & 655 & $\begin{array}{l}\text { Tremelimumab } 15 \mathrm{mg} / \mathrm{kg} \text { vs. } \\
\text { Chemotherapy }\end{array}$ & $\begin{array}{l}\text { CR; PR; ORR; AAE; Fatigue; } \\
\text { Pruritus; Rash; Diarrhea; Nausea }\end{array}$ \\
\hline Millward, 2013 & - & IV & 15 & $\begin{array}{l}\text { Tremelimumab } 6 \mathrm{mg} / \mathrm{kg} \text { vs. } \\
\text { Tremelimumab } 10 \mathrm{mg} / \mathrm{kg} \text { vs. } \\
\text { Tremelimumab } 15 \mathrm{mg} / \mathrm{kg}\end{array}$ & $\begin{array}{l}\text { AAE; Fatigue; Pruritus; Rash; } \\
\text { Diarrhea; Nausea }\end{array}$ \\
\hline Robert, 2011 & NCT00324155 & III/IV & 502 & $\begin{array}{l}\text { Ipilimumab } 10 \mathrm{mg} / \\
\text { kg+Chemotherapy vs. } \\
\text { Chemotherapy }\end{array}$ & $\begin{array}{l}\text { CR; PR; SD; PD; ORR; AAE; } \\
\text { Pruritus; Rash; Diarrhea }\end{array}$ \\
\hline Hersh, 2011 & NCT00050102 & II & 76 & $\begin{array}{l}\text { Ipilimumab } 3 \mathrm{mg} / \mathrm{kg} \text { vs. } \\
\text { Ipilimumab+Chemotherapy }\end{array}$ & $\begin{array}{l}\text { PD; ORR; AAE; Fatigue; } \\
\text { Pruritus; Rash; Diarrhea; Nausea }\end{array}$ \\
\hline Hamid, 2011 & NCT00261365 & II & 82 & $\begin{array}{l}\text { Ipilimumab } 3 \text { mg/kg vs. } \\
\text { Ipilimumab } 10 \mathrm{mg} / \mathrm{kg}\end{array}$ & CR; PR; SD; ORR \\
\hline Wolchok, 2011 & NCT00289640 & III/IV & 145 & $\begin{array}{l}\text { Ipilimumab } 10 \mathrm{mg} / \mathrm{kg} \text { vs. } \\
\text { Ipilimumab } 3 \mathrm{mg} / \mathrm{kg}\end{array}$ & $\begin{array}{l}\text { CR; PR; SD; PD; ORR; AAE; } \\
\text { Fatigue; Pruritus; Rash; Diarrhea; } \\
\text { Nausea }\end{array}$ \\
\hline Weber, 2009 & - & III/IV & 115 & $\begin{array}{l}\text { Ipilimumab } 10 \mathrm{mg} / \\
\mathrm{kg}+\text { Chemotherapy vs. } \\
\text { Ipilimumab } 10 \mathrm{mg} / \mathrm{kg}\end{array}$ & CR; PR; SD; PD; ORR; Diarrhea \\
\hline Camacho, 2009 & NCT0086489 & III & 115 & $\begin{array}{l}\text { Tremelimumab } 6 \mathrm{mg} / \mathrm{kg} \text { vs. } \\
\text { Tremelimumab } 10 \mathrm{mg} / \mathrm{kg} \text { vs. } \\
\text { Tremelimumab } 15 \mathrm{mg} / \mathrm{kg}\end{array}$ & $\begin{array}{l}\text { CR; PR; AAE; Fatigue; Pruritus; } \\
\text { Rash; Diarrhea; Nausea }\end{array}$ \\
\hline Ribas, 2005 & - & I & 20 & $\begin{array}{l}\text { Ipilimumab } 3 \mathrm{mg} / \mathrm{kg} \text { vs. } \\
\text { Ipilimumab } 10 \mathrm{mg} / \mathrm{kg}\end{array}$ & Fatigue; Diarrhea; Nausea \\
\hline
\end{tabular}

CR complete response; PR partial response; SD stable disease; PD progressive disease; ORR overall response rate; AAE all adverse events. 
Table 2: Meta-analysis results for pair-wise comparisons of clinical outcome

\begin{tabular}{|c|c|c|c|c|c|}
\hline Comparisons & $\begin{array}{l}\text { Complete } \\
\text { Response }\end{array}$ & Partial Response & Stable Disease & $\begin{array}{l}\text { Progressive } \\
\text { Disease }\end{array}$ & $\begin{array}{c}\text { Overall } \\
\text { Response Rate }\end{array}$ \\
\hline $\begin{array}{l}\text { Tremelimumab } 15 \mathrm{mg} / \mathrm{kg} \\
\text { vs. Chemotherapy }\end{array}$ & $1.37(0.54,3.45)$ & $1.04(0.58,1.85)$ & - & - & $1.12(0.68,1.85)$ \\
\hline $\begin{array}{l}\text { Nivolumab } 3 \mathrm{mg} / \mathrm{kg} \text { vs. } \\
\text { Chemotherapy }\end{array}$ & $6.51(1.95,21.76)$ & $2.57(1.76,3.75)$ & $0.83(0.59,1.16)$ & $0.85(0.53,1.38)$ & $2.92(2.02,4.21)$ \\
\hline $\begin{array}{l}\text { Pembrolizumab } 10 \mathrm{mg} / \mathrm{kg} \\
\text { vs. Chemotherapy }\end{array}$ & $6.18(2.11,18.12)$ & $5.07(2.31,11.14)$ & $0.93(0.55,1.58)$ & $0.77(0.54,1.09)$ & - \\
\hline $\begin{array}{l}\text { Pembrolizumab } 2 \mathrm{mg} / \mathrm{kg} \text { vs. } \\
\text { Chemotherapy }\end{array}$ & $5.22(1.76,15.51)$ & $4.23(1.90,9.38)$ & $0.96(0.57,1.64)$ & $0.75(0.53,1.07)$ & - \\
\hline $\begin{array}{l}\text { Ipilimumab+Chemotherapy } \\
\text { vs. Chemotherapy }\end{array}$ & $2.34(0.60,9.14)$ & $1.65(1.03,2.65)$ & $0.98(0.66,1.46)$ & $1.13(0.46,2.74)$ & - \\
\hline $\begin{array}{l}\text { Ipilimumab } 10 \mathrm{mg} / \mathrm{kg} \text { vs. } \\
\text { Ipilimumab } 3 \mathrm{mg} / \mathrm{kg}\end{array}$ & $1.62(0.37,7.08)$ & $0.75(0.31,1.81)$ & $0.62(0.19,2.01)$ & $1.02(0.561 .87)$ & $0.82(0.232 .98)$ \\
\hline $\begin{array}{l}\text { Nivolumab } 3 \mathrm{mg} / \mathrm{kg} \text { vs. } \\
\text { Ipilimumab } 3 \mathrm{mg} / \mathrm{kg}\end{array}$ & $3.99(1.72,9.26)$ & $2.07(1.449,2.97)$ & $0.49(0.32,0.76)$ & $0.77(0.581 .02)$ & $2.29(1.63,3.22)$ \\
\hline $\begin{array}{l}\text { Ipilimumab+Nivolumab vs. } \\
\text { Ipilimumab } 3 \mathrm{mg} / \mathrm{kg}\end{array}$ & $5.52(2.57,11.87)$ & $2.81(2.01,3.93)$ & $0.55(0.37,0.80)$ & $0.45(0.33,0.60)$ & $3.29(2.17,5.00)$ \\
\hline $\begin{array}{l}\text { Ipilimumab+Chemotherapy } \\
\text { vs. Ipilimumab } 3 \mathrm{mg} / \mathrm{kg}\end{array}$ & $1.70(0.26,11.07)$ & $1.02(0.54,1.95)$ & $1.23(0.73,2.10)$ & $1.08(0.73,1.58)$ & $1.21(0.65,2.26)$ \\
\hline $\begin{array}{l}\text { Ipilimumab+Chemotherapy } \\
\text { vs. Ipilimumab } 10 \mathrm{mg} / \mathrm{kg}\end{array}$ & $1.00(0.06,16.37)$ & $0.65(0.22,1.96)$ & $0.98(0.39,2.45)$ & $1.15(0.62,2.13)$ & $0.76(0.27,2.19)$ \\
\hline $\begin{array}{l}\text { Tremelimumab } 15 \mathrm{mg} / \mathrm{kg} \\
\text { vs. Tremelimumab } 10 \mathrm{mg} / \mathrm{kg}\end{array}$ & $2.48(1.64,3.76)$ & $0.96(0.18,4.99)$ & $1.12(0.46,2.68$ & $0.96(0.48,1.91)$ & $0.96(0.22,4.06)$ \\
\hline $\begin{array}{l}\text { Ipilimumab+Nivolumab vs. } \\
\text { Nivolumab } 3 \mathrm{mg} / \mathrm{kg}\end{array}$ & $1.29(0.77,2.17)$ & $1.33(0.99,1.78)$ & $1.21(0.75,1.96)$ & $0.60(0.43,0.84)$ & $1.32(1.01,1.73)$ \\
\hline $\begin{array}{l}\text { Pembrolizumab } 2 \mathrm{mg} / \mathrm{kg} \text { vs. } \\
\text { Pembrolizumab } 10 \mathrm{mg} / \mathrm{kg}\end{array}$ & $0.85(0.46,1.55)$ & $0.88(0.59,1.33)$ & $1.04(0.68,1.59)$ & $0.94(0.69,1.28)$ & $1.03(0.53,2.00)$ \\
\hline
\end{tabular}
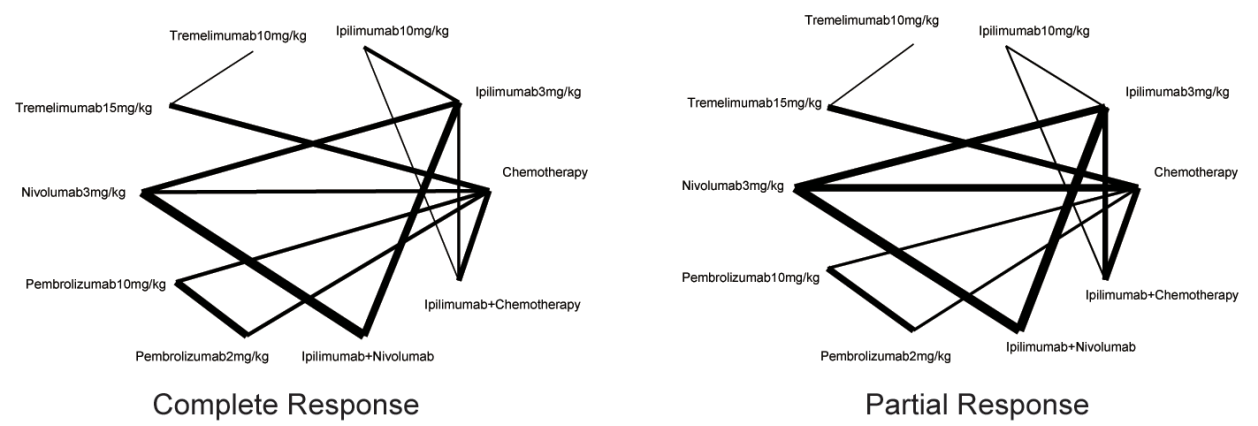

Partial Response
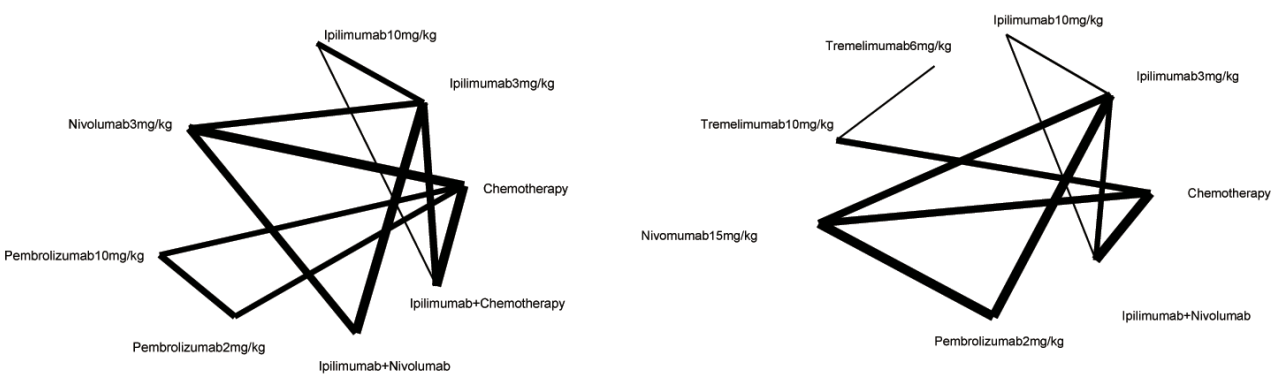

Stable Disease \& Progressive Disease

Overall Response Rate

Figure 1: Evidence network of eligible comparisons for complete response, partial response, stable \& progressive disease and overall response rate in network meta-analysis. The width of the lines represents the cumulative number of trials for each comparison and the size of every node is proportional to the number of enrolled participants (sample size). 
0.55, 95\% CI: 0.36-0.8, respectively; diarrhea: $\mathrm{OR}=0.58$, 95\% CI: $0.41-0.83$, OR $=0.64,95 \%$ CI: $0.41-0.98$, respectively). The combined application of ipilimumab and nivolumab could significantly attenuate the symptoms of diarrhea in ipilimumab treatment $(\mathrm{OR}=1.33$; $95 \%$ CI: 1.01-1.75), as well as decreased nausea and rash in nivolumab treatment $(\mathrm{OR}=1.99,95 \% \mathrm{CI}$ : 1.32-2.99; $\mathrm{OR}=1.56,95 \% \mathrm{CI}: 1.14-2.15)$.

Results from the network meta-analysis were illustrated in Figure 5 and Figure 6. Taking fatigue into account, ipilimumab $3 \mathrm{mg} / \mathrm{kg}$ and ipilimumab+

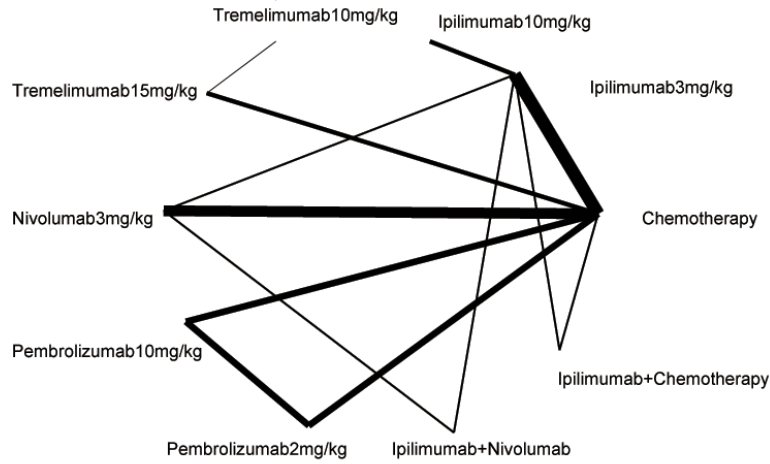

All Adverse Events

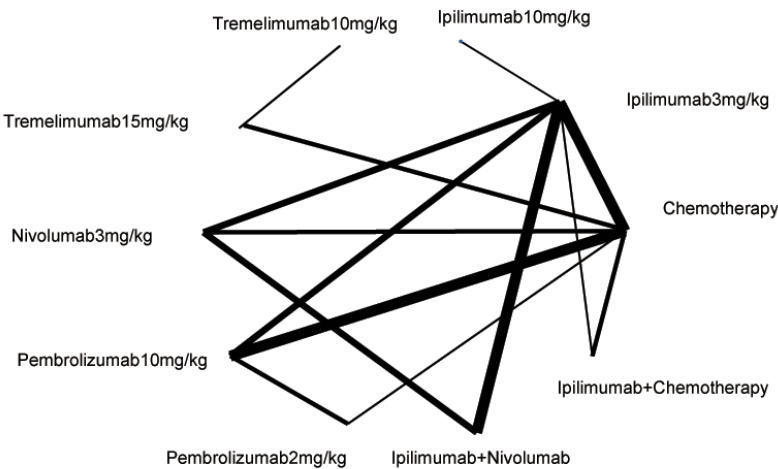

Pruritus

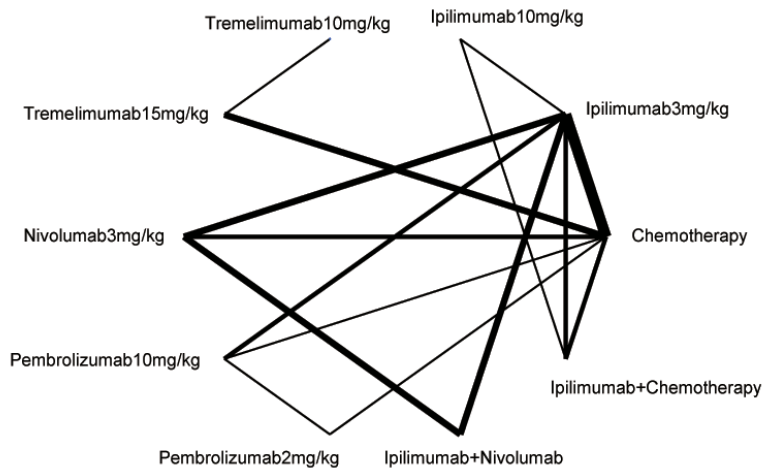

Diarrhea nivolumab triggered more fatigues than pembrolizumab $10 \mathrm{mg} / \mathrm{kg}, 2 \mathrm{mg} / \mathrm{kg}$ and tremelimumab $15 \mathrm{mg} / \mathrm{kg}$ (Table 5). Patients under ipilimumab+ chemotherapy were more likely to have fatigue compared to tremelimumab $15 \mathrm{mg} / \mathrm{kg}$, nivolumab $3 \mathrm{mg} / \mathrm{kg}$, pembrolizumab $10 \mathrm{mg} / \mathrm{kg}$, pembrolizumab $2 \mathrm{mg} / \mathrm{kg}$ and ipilimumab $3 \mathrm{mg} / \mathrm{kg}$. For all advert events, we found that pembrolizumab $2 \mathrm{mg} / \mathrm{kg}$ was less likely to cause advert events than ipilimumab + chemotherapy and ipilimumab + nivolumab. With respect to rash, Ipilimumab + nivolumab resulted in a significantly

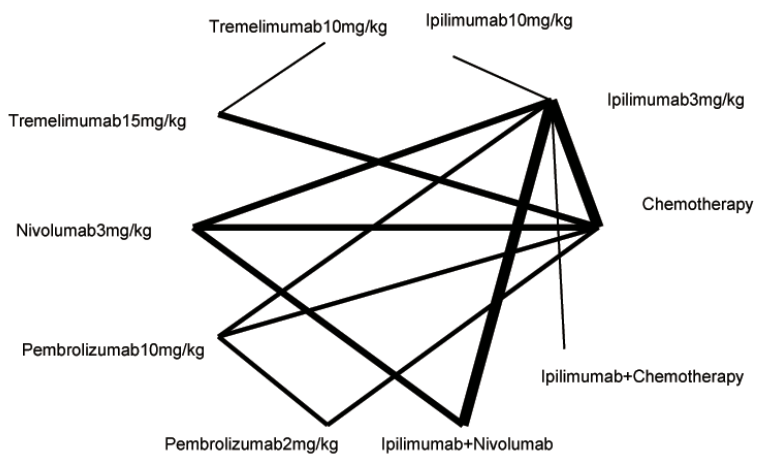

Fatigue

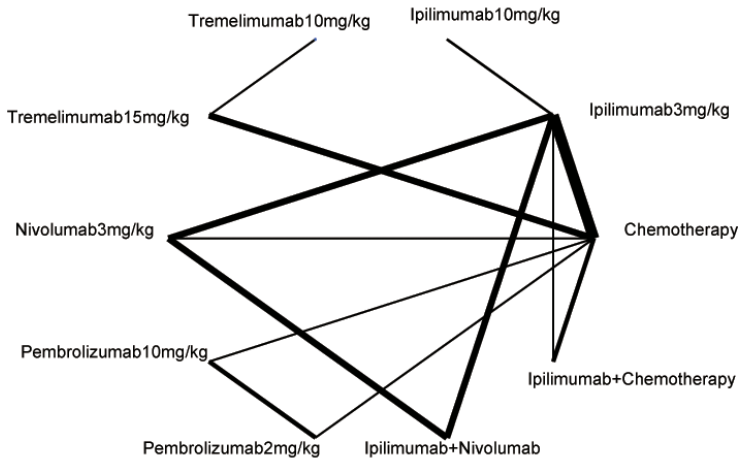

Rush

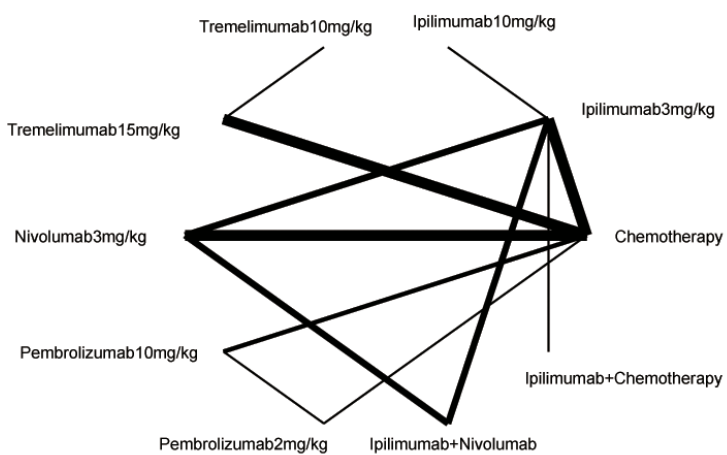

Nausea

Figure 2: Evidence network of eligible comparisons for various adverse events in network meta-analysis. The width of the lines represents the cumulative number of trials for each comparison and the size of every node is proportional to the number of enrolled participants (sample size). 
Table 3: Comparison of odds ratios of complete rate and partial rate for different interventions

\begin{tabular}{|c|c|c|c|c|c|c|c|c|c|}
\hline Chemotherapy & $\begin{array}{c}1.46 \\
(0.44,4.81)\end{array}$ & $\begin{array}{c}2.41 \\
(0.45,13.02)\end{array}$ & $\begin{array}{c}1.45(0.08 \\
27.70)\end{array}$ & $\begin{array}{c}1.38 \\
(0.55,3.49)\end{array}$ & $\begin{array}{c}6.74 \\
(2.32,19.55)\end{array}$ & $\begin{array}{c}6.99 \\
(2.38,20.49)\end{array}$ & $\begin{array}{c}5.80 \\
(1.95,17.21)\end{array}$ & $\begin{array}{c}9.09 \\
(2.88,28.75)\end{array}$ & $\begin{array}{c}2.42 \\
(0.76,7.68)\end{array}$ \\
\hline $\begin{array}{c}1.43 \\
(0.99,2.21)\end{array}$ & $\begin{array}{l}\text { Ipilimumab } \\
3 \mathrm{mg} / \mathrm{kg}\end{array}$ & $\begin{array}{c}1.66 \\
(0.44,6.28)\end{array}$ & $\begin{array}{c}0.99 \\
(0.04,24.03)\end{array}$ & $\begin{array}{c}0.95 \\
(0.21,4.31)\end{array}$ & $\begin{array}{c}4.63 \\
(2.16,9.95)\end{array}$ & $\begin{array}{c}4.80 \\
(0.96,23.99)\end{array}$ & $\begin{array}{c}3.98 \\
(0.79,20.07)\end{array}$ & $\begin{array}{c}6.25 \\
(2.97,13.13)\end{array}$ & $\begin{array}{c}1.66 \\
(0.47 .5 .83)\end{array}$ \\
\hline $\begin{array}{c}1.46 \\
(0.92,2.21)\end{array}$ & $\begin{array}{c}1.02 \\
(0.50,2.09)\end{array}$ & $\begin{array}{l}\text { Ipilimumab } \\
10 \mathrm{mg} / \mathrm{kg}\end{array}$ & $\begin{array}{c}0.60 \\
(0.02,17.94)\end{array}$ & $\begin{array}{c}0.57 \\
(0.08,3.91)\end{array}$ & $\begin{array}{c}2.79 \\
(0.62,12.48)\end{array}$ & $\begin{array}{c}2.89 \\
(0.39,21.37)\end{array}$ & $\begin{array}{c}2.40 \\
(0.32,17.85)\end{array}$ & $\begin{array}{c}3.77 \\
(0.84,16.82)\end{array}$ & $\begin{array}{c}1.00 \\
(0.20,5.12)\end{array}$ \\
\hline $\begin{array}{c}1.09 \\
(0.19,6.32)\end{array}$ & $\begin{array}{c}0.76 \\
(0.13,4.67)\end{array}$ & $\begin{array}{c}0.75 \\
(0.11,5.10)\end{array}$ & $\begin{array}{l}\text { Tremelimumab } \\
10 \mathrm{mg} / \mathrm{kg}\end{array}$ & $\begin{array}{c}0.96 \\
(0.06,15.76)\end{array}$ & $\begin{array}{c}4.66 \\
(0.20,107.30)\end{array}$ & $\begin{array}{c}4.83 \\
(0.21,111.68)\end{array}$ & $\begin{array}{c}4.00 \\
(0.17,93.04)\end{array}$ & $\begin{array}{c}6.28 \\
(0.26,149.22)\end{array}$ & $\begin{array}{c}1.67 \\
(0.07,39.74)\end{array}$ \\
\hline $\begin{array}{c}1.04 \\
(0.58,1.86)\end{array}$ & $\begin{array}{c}0.73 \\
(0.35,1.51)\end{array}$ & $\begin{array}{c}0.71 \\
(0.27,1.88)\end{array}$ & $\begin{array}{c}0.95 \\
(0.18,5.00)\end{array}$ & $\begin{array}{c}\text { Tremelimumab } \\
15 \mathrm{mg} / \mathrm{kg}\end{array}$ & $\begin{array}{c}4.87 \\
(1.19,19.95)\end{array}$ & $\begin{array}{c}5.05 \\
(1.22,20.86)\end{array}$ & $\begin{array}{c}4.19 \\
(1.01,17.47)\end{array}$ & $\begin{array}{c}6.57 \\
(1.50,28.76)\end{array}$ & $\begin{array}{c}1.75 \\
(0.40,7.67)\end{array}$ \\
\hline $\begin{array}{c}3.55 \\
(2.50,5.04)\end{array}$ & $\begin{array}{c}2.48 \\
(1.77,3.48)\end{array}$ & $\begin{array}{c}2.43 \\
(1.13,5.19)\end{array}$ & $\begin{array}{c}3.25 \\
(0.54,19.45)\end{array}$ & $\begin{array}{c}3.40 \\
(1.72,6.72)\end{array}$ & $\begin{array}{c}\text { Nivolumab } 3 \\
\mathrm{mg} / \mathrm{kg}\end{array}$ & $\begin{array}{c}1.04 \\
(0.23,4.71)\end{array}$ & $\begin{array}{c}0.86 \\
(0.19,3.94)\end{array}$ & $\begin{array}{c}1.35 \\
(0.81,2.26)\end{array}$ & $\begin{array}{c}0.36 \\
(0.10,1.29)\end{array}$ \\
\hline $\begin{array}{c}6.05 \\
(2.78,13.13)\end{array}$ & $\begin{array}{c}4.23 \\
(1.74,10.32)\end{array}$ & $\begin{array}{c}4.14 \\
(1.38,12.43)\end{array}$ & $\begin{array}{c}5.53 \\
(0.81,37.76)\end{array}$ & $\begin{array}{c}5.80 \\
(2.20,15.33)\end{array}$ & $\begin{array}{c}1.70 \\
(0.73,4.00)\end{array}$ & $\begin{array}{l}\text { Pembrolizuma } \\
10 \mathrm{mg} / \mathrm{kg}\end{array}$ & $\begin{array}{c}0.83 \\
(0.45,1.52)\end{array}$ & $\begin{array}{c}1.30 \\
(0.27,6.29)\end{array}$ & $\begin{array}{c}0.35 \\
(0.07,1.68)\end{array}$ \\
\hline $\begin{array}{c}5.18 \\
(2.36,11.36)\end{array}$ & $\begin{array}{c}3.63 \\
(1.48,8.90)\end{array}$ & $\begin{array}{c}3.54 \\
(1.17,10.71)\end{array}$ & $\begin{array}{c}4.74 \\
(0.69,32.46)\end{array}$ & $\begin{array}{c}4.97 \\
(1.87,13.22)\end{array}$ & $\begin{array}{c}1.46 \\
(0.62,3.45)\end{array}$ & $\begin{array}{c}0.86 \\
(0.57,1.30)\end{array}$ & $\begin{array}{c}\text { Pembrolizuma } 2 \\
\mathrm{mg} / \mathrm{kg}\end{array}$ & $\begin{array}{c}1.57 \\
(0.32,7.65)\end{array}$ & $\begin{array}{c}0.42 \\
(0.09,2.04)\end{array}$ \\
\hline $\begin{array}{c}5.92 \\
(3.78,9.27)\end{array}$ & $\begin{array}{c}4.14 \\
(2.95,5.83)\end{array}$ & $\begin{array}{c}4.05 \\
(1.87,8.78)\end{array}$ & $\begin{array}{c}5.42 \\
(0.89,33.19)\end{array}$ & $\begin{array}{c}5.68 \\
(2.73,11.85)\end{array}$ & $\begin{array}{c}1.67 \\
(1.22,2.28)\end{array}$ & $\begin{array}{c}0.98 \\
(0.40,2.40)\end{array}$ & $\begin{array}{c}1.14 \\
(0.46,2.82)\end{array}$ & $\begin{array}{l}\text { Ipilimumab+ } \\
\text { Nivolumab }\end{array}$ & $\begin{array}{c}0.27 \\
(0.07,1.00)\end{array}$ \\
\hline $\begin{array}{c}1.53 \\
(1.02,2.30)\end{array}$ & $\begin{array}{c}1.07 \\
(0.68,1.69)\end{array}$ & $\begin{array}{c}1.05 \\
(0.50,2.21)\end{array}$ & $\begin{array}{c}1.40 \\
(0.23,8.51)\end{array}$ & $\begin{array}{c}1.47 \\
(0.72,2.99)\end{array}$ & $\begin{array}{c}0.43 \\
(0.27,0.68)\end{array}$ & $\begin{array}{c}0.25 \\
(0.11,0.61)\end{array}$ & $\begin{array}{c}0.30 \\
(0.12,0.72)\end{array}$ & $\begin{array}{c}0.26 \\
(0.16,0.43)\end{array}$ & $\begin{array}{l}\text { Ipilimumab+ } \\
\text { Chemotherapy }\end{array}$ \\
\hline
\end{tabular}

Note: Odds ratios in the blue zone complete is for complete response and in the white zone for partial response. The column treatment is compared with the row treatment in blue squares while it is opposite in the white squares. Numbers in parentheses indicate $95 \%$ credible intervals.

Table 4: Meta-analysis results for pair-wise comparisons of adverse events

\begin{tabular}{|c|c|c|c|c|c|c|}
\hline Comparisons & $\begin{array}{l}\text { All adverse } \\
\text { events }\end{array}$ & Fatigue & Pruritus & Diarrhea & Nausea & Rash \\
\hline $\begin{array}{l}\text { Chemotherapy vs. } \\
\text { Ipilimumab } 3 \mathrm{mg} / \mathrm{kg}\end{array}$ & $1.08(0.90,1.30)$ & $1.32(1.03,1.70)$ & $2.91(2.15,3.92)$ & $1.63(1.27,2.08)$ & $1.41(1.03,1.91)$ & $2.32(1.73,3.10)$ \\
\hline $\begin{array}{l}\text { Chemotherapy vs. } \\
\text { Tremelimumab } 15 \mathrm{mg} / \mathrm{kg}\end{array}$ & $1.06(0.85,1.33)$ & $0.90(0.66,1.21)$ & $6.23(3.60,10.80)$ & $2.95(2.10,4.15)$ & $0.69(0.52,0.92)$ & $0.90(0.66,1.21)$ \\
\hline $\begin{array}{l}\text { Chemotherapy vs. Nivolumab } \\
3 \mathrm{mg} / \mathrm{kg}\end{array}$ & $1.02(0.83,1.27)$ & $1.11(0.77,1.59)$ & $4.86(1.56,15.11)$ & $0.99(0.66,1.49)$ & $0.36(0.26,0.51)$ & $5.12(2.09,12.52)$ \\
\hline $\begin{array}{l}\text { Chemotherapy vs. } \\
\text { Pembrolizumab } 10 \mathrm{mg} / \mathrm{kg}\end{array}$ & $0.95(0.69,1.30)$ & $0.83(0.54,1.27)$ & $6.92(2.87,16.69)$ & $1.34(0.65,2.76)$ & $0.28(0.15,0.52)$ & $2.22(0.94,5.25)$ \\
\hline $\begin{array}{l}\text { Chemotherapy vs. } \\
\text { Pembrolizumab } 2 \mathrm{mg} / \mathrm{kg}\end{array}$ & $0.86(0.63,1.19)$ & $0.64(0.41,1.00)$ & $6.13(2.53,14.89)$ & $1.06(0.50,2.27)$ & $0.15(0.07,0.33)$ & $2.61(1.13,6.05)$ \\
\hline $\begin{array}{l}\text { Chemotherapy vs. } \\
\text { Ipilimumab+Chemotherapy }\end{array}$ & $1.04(0.81,1.34)$ & - & $3.34(2.01,5.56)$ & $2.04(1.34,3.10)$ & - & $3.62(2.05,6.37)$ \\
\hline $\begin{array}{l}\text { Ipilimumab } 3 \mathrm{mg} / \mathrm{kg} \text { vs. } \\
\text { Ipilimumab } 10 \mathrm{mg} / \mathrm{kg}\end{array}$ & $0.73(0.40,1.34)$ & $1.24(0.58,2.66)$ & $0.13(0.03,0.60)$ & $0.71(0.33,1.53)$ & $0.83(0.35,1.98)$ & $0.17(0.05,0.62)$ \\
\hline $\begin{array}{l}\text { Ipilimumab } 3 \mathrm{mg} / \mathrm{kg} \text { vs. } \\
\text { Nivolumab } 3 \mathrm{mg} / \mathrm{kg}\end{array}$ & $1.01(0.81,1.26)$ & $1.23(0.89,1.69)$ & $0.53(0.38,0.76)$ & $0.58(0.41,0.83)$ & $0.82(0.53,1.27)$ & $0.79(0.57,1.10)$ \\
\hline $\begin{array}{l}\text { Ipilimumab } 3 \mathrm{mg} / \mathrm{kg} \text { vs. } \\
\text { Pembrolizumab } 10 \mathrm{mg} / \mathrm{kg}\end{array}$ & - & $0.92(0.61,1.38)$ & $0.55(0.36,0.84)$ & $0.64(0.41,0.98)$ & - & - \\
\hline $\begin{array}{l}\text { Ipilimumab } 3 \mathrm{mg} / \mathrm{kg} \text { vs. } \\
\text { Ipilimumab+Nivolumab }\end{array}$ & $1.02(0.83,1.25)$ & $1.20(0.90,1.61)$ & $0.99(0.74,1.32)$ & $1.33(1.01,1.75)$ & $1.45(0.96,2.12)$ & $1.29(0.97,1.71)$ \\
\hline $\begin{array}{l}\text { Ipilimumab } 3 \mathrm{mg} / \mathrm{kg} \text { vs. } \\
\text { Ipilimumab+Chemotherapy }\end{array}$ & $1.19(0.60,2.34)$ & $1.83(0.88,3.82)$ & $0.66(0.28,1.54)$ & $1.03(0.56,1.89)$ & $1.50(0.61,3.67)$ & $0.91(0.47,1.76)$ \\
\hline $\begin{array}{l}\text { Ipilimumab } 10 \mathrm{mg} / \mathrm{kg} \text { vs. } \\
\text { Ipilimumab+Chemotherapy }\end{array}$ & - & - & - & $1.38(0.41,4.56)$ & - & - \\
\hline $\begin{array}{l}\text { Tremelimumab } 10 \mathrm{mg} / \mathrm{kg} \text { vs. } \\
\text { Tremelimumab } 15 \mathrm{mg} / \mathrm{kg}\end{array}$ & $1.00(0.19,5.36)$ & $0.79(0.31,1.99)$ & $1.18(0.42,3.35)$ & $1.11(0.54,2.30)$ & $0.89(0.37,2.15)$ & $0.97(0.45,2.07)$ \\
\hline $\begin{array}{l}\text { Tremelimumab } 15 \mathrm{mg} / \mathrm{kg} \text { vs. } \\
\text { Ipilimumab+Nivolumab }\end{array}$ & - & - & $1.77(1.24,2.53)$ & $2.32(1.65,3.25)$ & - & - \\
\hline $\begin{array}{l}\text { Nivolumab } 3 \mathrm{mg} / \mathrm{kg} \text { vs. } \\
\text { Ipilimumab+Nivolumab }\end{array}$ & $1.01(0.81,1.26)$ & $1.03(0.76,1.41)$ & - & - & $1.99(1.32,2.99)$ & $1.56(1.14,2.15)$ \\
\hline $\begin{array}{l}\text { Pembrolizumab } 10 \mathrm{mg} / \mathrm{kg} \text { vs. } \\
\text { Pembrolizumab } 2 \mathrm{mg} / \mathrm{kg}\end{array}$ & $0.91(0.66,1.26)$ & $1.39(0.26,7.40)$ & $0.88(0.54,1.44)$ & $0.8(0.44,1.59)$ & $0.54(0.22,1.29)$ & $1.16(0.61,2.21)$ \\
\hline
\end{tabular}




\section{Treatment Effect Complete Response}

\section{Compared with Chemotherapy}

Ipilimumab $3 \mathrm{mg} / \mathrm{kg}$ Ipilimumab $10 \mathrm{mg} / \mathrm{kg}$

Tremelimumab $10 \mathrm{mg} / \mathrm{kg}$

Tremelimumab $15 \mathrm{mg} / \mathrm{kg}$

Nivolumab $3 \mathrm{mg} / \mathrm{kg}$

Pembrolizumab $10 \mathrm{mg} / \mathrm{kg}$

Pembrolizumab 2mg/kg

Ipilimumab+Nivolumab

Ipilimumab+Chemotherapy

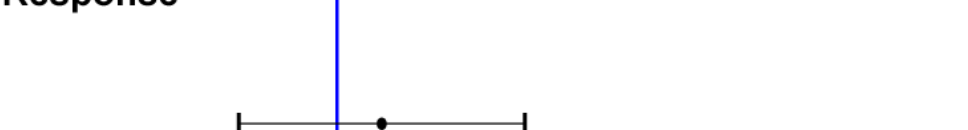

OR with $95 \% \mathrm{Cl}$

Compared with Ipilimumab $3 \mathrm{mg} / \mathrm{kg}$

Ipilimumab $10 \mathrm{mg} / \mathrm{kg}$

Tremelimumab $10 \mathrm{mg} / \mathrm{kg}$

Tremelimumab $15 \mathrm{mg} / \mathrm{kg}$

Nivolumab $3 \mathrm{mg} / \mathrm{kg}$

Pembrolizumab $10 \mathrm{mg} / \mathrm{kg}$

Pembrolizumab $2 \mathrm{mg} / \mathrm{kg}$

Ipilimumab+Nivolumab

Ipilimumab+Chemotherapy

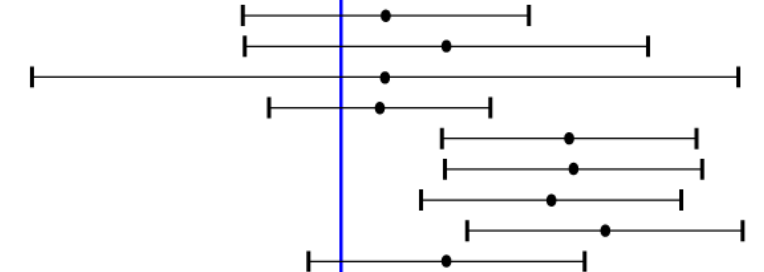

$1.46(0.44,4.81)$

$2.41(0.45,13.02)$

$1.45(0.08,27.70)$

$1.38(0.55,3.49)$

$6.74(2.32,19.55)$

$6.99(2.38,20.49)$

$5.80(1.95,17.21)$

$9.09(2.88,28.75)$

$2.42(0.76,7.68)$

$1.66(0.44,6.28)$

$0.99(0.04,24.03)$

$0.95(0.21,4.31)$

$4.63(2.16,9.95)$

$4.80(0.96,23.99)$

$3.98(0.79,20.07)$

$6.25(2.97,13.13)$

$1.66(0.47,5.83)$

\section{Compared with Ipilimumab $10 \mathrm{mg} / \mathrm{kg}$}

Tremelimumab $10 \mathrm{mg} / \mathrm{kg}$

Tremelimumab $15 \mathrm{mg} / \mathrm{kg}$

Nivolumab $3 \mathrm{mg} / \mathrm{kg}$

Pembrolizumab $10 \mathrm{mg} / \mathrm{kg}$

Pembrolizumab 2mg/kg

Ipilimumab+Nivolumab

Ipilimumab+Chemotherapy
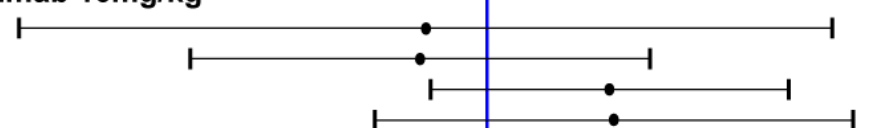

$0.60(0.02,17.94)$

$0.57(0.08,3.91)$

$2.79(0.62,12.48)$

$2.89(0.39,21.37)$

$2.40(0.32,17.85)$

$3.77(0.84,16.82)$

$1.00(0.20,5.12)$

Compared with Tremelimumab $10 \mathrm{mg} / \mathrm{kg}$

Tremelimumab $15 \mathrm{mg} / \mathrm{kg}$

Nivolumab $3 \mathrm{mg} / \mathrm{kg}$

Pembrolizumab $10 \mathrm{mg} / \mathrm{kg}$

Pembrolizumab $2 \mathrm{mg} / \mathrm{kg}$

Ipilimumab+Nivolumab

Ipilimumab+Chemotherapy

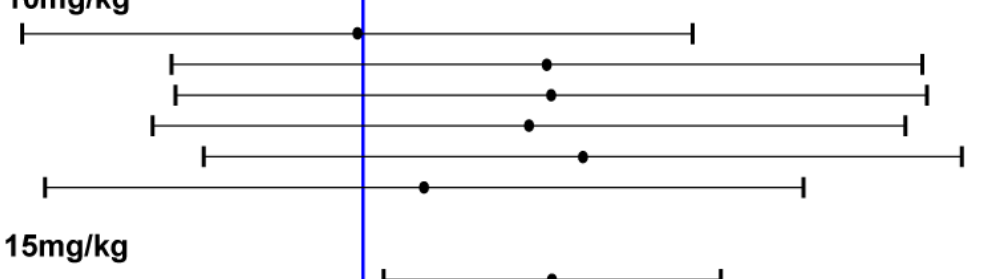

$0.96(0.06,15.76)$

$4.66(0.20,107.30)$

$4.83(0.21,111.68)$

$4.00(0.17,93.04)$

$6.28(0.26,149.22)$

$1.67(0.07,39.74)$

Compared with Tremelimumab $15 \mathrm{mg} / \mathrm{kg}$

Nivolumab $3 \mathrm{mg} / \mathrm{kg}$

Pembrolizumab $10 \mathrm{mg} / \mathrm{kg}$

Pembrolizumab $2 \mathrm{mg} / \mathrm{kg}$

Ipilimumab+Nivolumab

Ipilimumab+Chemotherapy

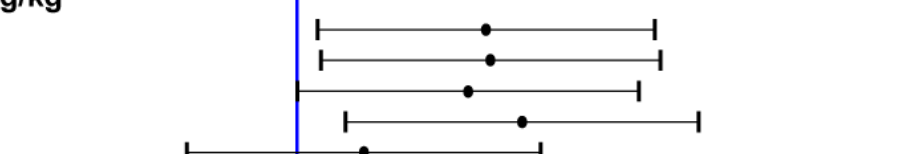

$4.87(1.19,19.95)$

$5.05(1.22,20.86)$

$4.19(1.01,17.47)$

$6.57(1.50,28.76)$

$1.75(0.40,7.67)$

Compared with Nivolumab $3 \mathrm{mg} / \mathrm{kg}$

Pembrolizumab $10 \mathrm{mg} / \mathrm{kg}$

Pembrolizumab $2 \mathrm{mg} / \mathrm{kg}$

Ipilimumab+Nivolumab

Ipilimumab+Chemotherapy

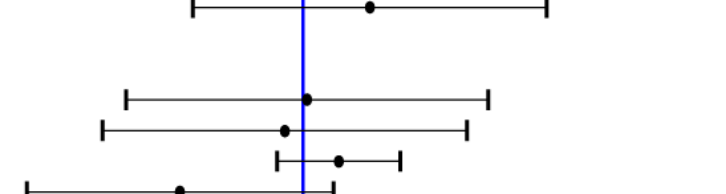

$1.04(0.23,4.71)$

$0.86(0.19,3.94)$

$1.35(0.81,2.26)$

$0.36(0.10,1.29)$

Compared with Pembrolizumab $10 \mathrm{mg} / \mathrm{kg}$

Pembrolizumab 2mg/kg

Ipilimumab+Nivolumab

Ipilimumab+Chemotherapy

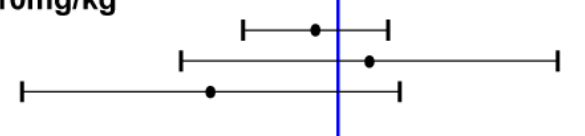

$0.83(0.45,1.52)$

$1.30(0.27,6.29)$

$0.35(0.07,1.68)$

Compared with Pembrolizumab 2mg/kg

Ipilimumab+Nivolumab

Ipilimumab+Chemotherapy

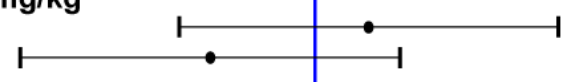

$1.57(0.32,7.65)$

$0.42(0.09,2.04)$

Compared with Ipilimumab+Nivolumab

Ipilimumab+Chemotherapy

$0.27(0.07,1.00)$

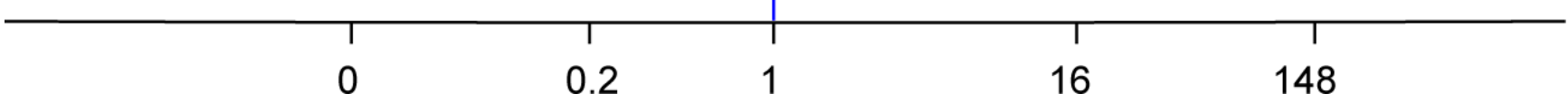

Figure 3: Forest plots for the correlations of the complete-response outcome of 10 interventions on melanoma. 
higher risk of rash than chemotherapy, ipilimumab $3 \mathrm{mg} / \mathrm{kg}$, ipilimumab $10 \mathrm{mg} / \mathrm{kg}$, tremelimumab $10 \mathrm{mg} / \mathrm{kg}$, tremelimumab $15 \mathrm{mg} / \mathrm{kg}$ and nivolumab 3 $\mathrm{mg} / \mathrm{kg}$ (Table 6). And Ipilimumab $3 \mathrm{mg} / \mathrm{kg}$, nivolumab $3 \mathrm{mg} / \mathrm{kg}$, and ipilimumab + chemotherapy treatment resulted in higher possibilities of rash than ipilimumab $10 \mathrm{mg} / \mathrm{kg}$, tremelimumab $10 \mathrm{mg} / \mathrm{kg}$ and $15 \mathrm{mg} / \mathrm{kg}$. In addition, tremelimumab $15 \mathrm{mg} / \mathrm{kg}$ had a significant higher possibility of diarrhea than all therapies except
Tremelimumab $10 \mathrm{mg} / \mathrm{kg}$ and Ipilimumab + Nivolumab (Table 6).

\section{Cumulative analysis and publication bias}

We generated the SUCRA curve to calculate and rank the cumulative probability of all treatments and outcomes. The results are presented in Table 7, Figure 7 and Figure 8 . We observed that chemotherapy ranked

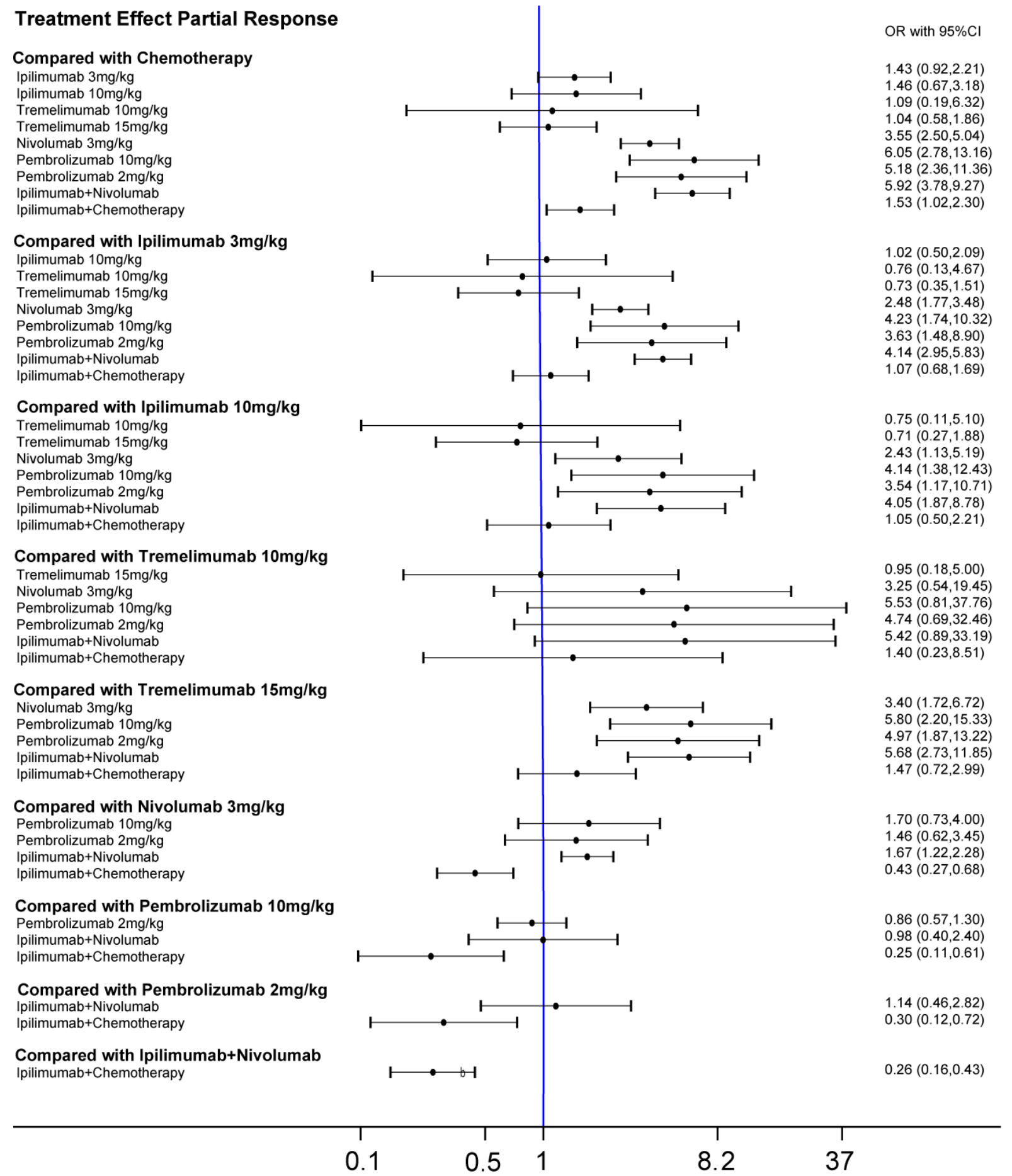

Figure 4: Forest plots for the correlations of the partial-response outcome of 10 interventions on melanoma. 
Table 5: Comparison of odds ratios of adverse events and fatigue for different interventions

\begin{tabular}{|c|c|c|c|c|c|c|c|c|c|}
\hline Chemotherapy & $\begin{array}{c}2.20 \\
(0.83,5.84)\end{array}$ & $\begin{array}{c}1.29 \\
(0.25,6.56)\end{array}$ & $\begin{array}{c}2.34 \\
(0.07,75.84)\end{array}$ & $\begin{array}{c}2.34 \\
(0.65,8.39)\end{array}$ & $\begin{array}{c}1.32 \\
(0.58,2.97)\end{array}$ & $\begin{array}{c}0.80 \\
(0.24,2.71)\end{array}$ & $\begin{array}{c}0.59 \\
(0.18,2.00)\end{array}$ & $\begin{array}{c}5.00 \\
(0.89,28.05)\end{array}$ & $\begin{array}{c}3.66 \\
(1.13,11.86)\end{array}$ \\
\hline $\begin{array}{c}1.54 \\
(1.08,2.20)\end{array}$ & $\begin{array}{l}\text { Ipilimumab } \\
3 \mathrm{mg} / \mathrm{kg}\end{array}$ & $\begin{array}{c}0.58 \\
(0.16,2.15)\end{array}$ & $\begin{array}{c}1.06 \\
(0.03,39.45)\end{array}$ & $\begin{array}{c}1.06 \\
(0.21,5.31)\end{array}$ & $\begin{array}{c}0.60 \\
(0.20,1.79)\end{array}$ & $\begin{array}{c}0.36 \\
(0.08,1.74)\end{array}$ & $\begin{array}{c}0.27 \\
(0.06,1.28)\end{array}$ & $\begin{array}{c}2.28 \\
(0.47,11.07)\end{array}$ & $\begin{array}{c}1.67 \\
(0.49,5.64)\end{array}$ \\
\hline $\begin{array}{c}2.00 \\
(0.83,4.82)\end{array}$ & $\begin{array}{c}1.30 \\
(0.58,2.91)\end{array}$ & $\begin{array}{l}\text { Ipilimumab } \\
10 \mathrm{mg} / \mathrm{kg}\end{array}$ & $\begin{array}{c}1.82 \\
(0.04,84.75)\end{array}$ & $\begin{array}{c}1.82 \\
(0.23,14.42)\end{array}$ & $\begin{array}{c}1.02 \\
(0.19,5.61)\end{array}$ & $\begin{array}{c}0.62 \\
(0.08,4.76)\end{array}$ & $\begin{array}{c}0.46 \\
(0.06,3.53)\end{array}$ & $\begin{array}{c}3.89 \\
(0.50,30.24)\end{array}$ & $\begin{array}{c}2.85 \\
(0.48,16.98)\end{array}$ \\
\hline $\begin{array}{c}1.14 \\
(0.40,3.27)\end{array}$ & $\begin{array}{c}0.74 \\
(0.25,2.25)\end{array}$ & $\begin{array}{c}0.57 \\
(0.15,2.25)\end{array}$ & $\begin{array}{l}\text { Tremelimumab } \\
10 \mathrm{mg} / \mathrm{kg}\end{array}$ & $\begin{array}{c}1.00 \\
(0.04,25.46)\end{array}$ & $\begin{array}{c}0.56 \\
(0.02,20.10)\end{array}$ & $\begin{array}{c}0.34 \\
(0.01,13.68)\end{array}$ & $\begin{array}{c}0.25 \\
(0.01,10.14)\end{array}$ & $\begin{array}{c}2.14 \\
(0.04,104.05)\end{array}$ & $\begin{array}{c}1.57 \\
(0.04,61.70)\end{array}$ \\
\hline $\begin{array}{c}0.85 \\
(0.57,1.26)\end{array}$ & $\begin{array}{c}0.55 \\
(0.32,0.94)\end{array}$ & $\begin{array}{c}0.42 \\
(0.16,1.11)\end{array}$ & $\begin{array}{c}0.74 \\
(0.26,1.95)\end{array}$ & $\begin{array}{l}\text { Tremelimumab } \\
\qquad 15 \mathrm{mg} / \mathrm{kg}\end{array}$ & $\begin{array}{c}0.56 \\
(0.12,2.57)\end{array}$ & $\begin{array}{c}0.34 \\
(0.06 .2 .00)\end{array}$ & $\begin{array}{c}0.25 \\
(0.04,1.48)\end{array}$ & $\begin{array}{c}2.14 \\
(0.25,18.30)\end{array}$ & $\begin{array}{c}1.57 \\
(0.28,8.89)\end{array}$ \\
\hline $\begin{array}{c}1.83 \\
(0.94,3.57)\end{array}$ & $\begin{array}{c}0.73 \\
(0.43,1.24)\end{array}$ & $\begin{array}{c}0.57 \\
(0.22,1.49)\end{array}$ & $\begin{array}{c}0.99 \\
(0.32,3.03)\end{array}$ & $\begin{array}{c}1.34 \\
(0.77,1.27)\end{array}$ & $\begin{array}{c}\text { Nivolumab } 3 \\
\mathrm{mg} / \mathrm{kg}\end{array}$ & $\begin{array}{c}0.61 \\
(0.14,2.63)\end{array}$ & $\begin{array}{c}0.45 \\
(0.10,1.95)\end{array}$ & $\begin{array}{c}3.80 \\
(0.69,20.91)\end{array}$ & $\begin{array}{c}2.78 \\
(0.71,10.81)\end{array}$ \\
\hline $\begin{array}{c}1.13 \\
(0.77,1.67)\end{array}$ & $\begin{array}{c}0.49 \\
(0.27,0.92)\end{array}$ & $\begin{array}{c}0.38 \\
(0.14,1.05)\end{array}$ & $\begin{array}{c}0.67 \\
(0.21,2.13)\end{array}$ & $\begin{array}{c}0.90 \\
(0.47,1.71)\end{array}$ & $\begin{array}{c}0.67 \\
(0.36,1.27)\end{array}$ & $\begin{array}{l}\text { Pembrolizuma } \\
10 \mathrm{mg} / \mathrm{kg}\end{array}$ & $\begin{array}{c}0.74 \\
(0.22,2.49)\end{array}$ & $\begin{array}{c}6.25 \\
(0.76,51.65)\end{array}$ & $\begin{array}{c}4.58 \\
(0.84,24.88)\end{array}$ \\
\hline $\begin{array}{c}1.82 \\
(0.84,3.96)\end{array}$ & $\begin{array}{c}0.35 \\
(0.19,0.66)\end{array}$ & $\begin{array}{c}0.27 \\
(0.10,0.75)\end{array}$ & $\begin{array}{c}0.47 \\
(0.15,1.52)\end{array}$ & $\begin{array}{c}0.64 \\
(0.33,1.23)\end{array}$ & $\begin{array}{c}0.48 \\
(0.25,0.91)\end{array}$ & $\begin{array}{c}0.71 \\
(0.42,1.21)\end{array}$ & $\begin{array}{c}\text { Pembrolizuma } 2 \\
\mathrm{mg} / \mathrm{kg}\end{array}$ & $\begin{array}{c}8.42 \\
(1.02,69.38)\end{array}$ & $\begin{array}{c}6.17 \\
(1.14,33.40)\end{array}$ \\
\hline $\begin{array}{c}0.76 \\
(0.46,1.26)\end{array}$ & $\begin{array}{c}1.41 \\
(0.94,2.12)\end{array}$ & $\begin{array}{c}1.09 \\
(0.44,2.69)\end{array}$ & $\begin{array}{c}1.90 \\
(0.58,6.21)\end{array}$ & $\begin{array}{c}2.57 \\
(1.31,5.05)\end{array}$ & $\begin{array}{c}1.92 \\
(0.99,3.75)\end{array}$ & $\begin{array}{c}2.86 \\
(1.36,6.00)\end{array}$ & $\begin{array}{c}4.03 \\
(1.90,8.58)\end{array}$ & $\begin{array}{l}\text { Ipilimumab+ } \\
\text { Nivolumab }\end{array}$ & $\begin{array}{c}0.73 \\
(0.11,5.03)\end{array}$ \\
\hline $\begin{array}{c}7.05 \\
(0.75,66.50)\end{array}$ & $\begin{array}{c}2.30 \\
(1.05,5.04)\end{array}$ & $\begin{array}{c}1.77 \\
(0.57,5.45)\end{array}$ & $\begin{array}{c}3.09 \\
(0.79,12.03)\end{array}$ & $\begin{array}{c}4.18 \\
(1.61,10.82)\end{array}$ & $\begin{array}{c}3.13 \\
(1.21,8.07)\end{array}$ & $\begin{array}{c}4.65 \\
(1.71,12.62)\end{array}$ & $\begin{array}{c}6.56 \\
(2.39,17.89)\end{array}$ & $\begin{array}{c}1.63 \\
(0.67,3.95)\end{array}$ & $\begin{array}{l}\text { Ipilimumab+ } \\
\text { Chemotherapy }\end{array}$ \\
\hline
\end{tabular}

Note: Odds ratios in the blue zone is for all adverse events and in the white zone for fatigue. The column treatment is compared with the row treatment in blue squares while it is opposite in the white squares. Numbers in parentheses indicate $95 \%$ credible intervals.

\section{Table 6: Comparison of odds ratios of diarrhea and rash for different interventions}

\begin{tabular}{|c|c|c|c|c|c|c|c|c|c|}
\hline Chemotherapy & $\begin{array}{c}2.23 \\
(1.79,2.78)\end{array}$ & $\begin{array}{c}1.53 \\
(0.77,3.03)\end{array}$ & $\begin{array}{c}4.12 \\
(1.71,9.91)\end{array}$ & $\begin{array}{c}4.96 \\
(3.46,7.11)\end{array}$ & $\begin{array}{c}1.04 \\
(0.77,1.40)\end{array}$ & $\begin{array}{c}1.31 \\
(0.87,1.97)\end{array}$ & $\begin{array}{c}1.05 \\
(0.54,2.04)\end{array}$ & $\begin{array}{c}3.56 \\
(2.53,5.02)\end{array}$ & $\begin{array}{c}2.44 \\
(1.72,3.47)\end{array}$ \\
\hline $\begin{array}{c}3.91 \\
(2.42,6.32)\end{array}$ & $\begin{array}{c}\text { Ipilimumab } \\
3 \mathrm{mg} / \mathrm{kg}\end{array}$ & $\begin{array}{c}0.68 \\
(0.35,1.33)\end{array}$ & $\begin{array}{c}1.85 \\
(0.75,4.56)\end{array}$ & $\begin{array}{c}2.22 \\
(1.46,3.39)\end{array}$ & $\begin{array}{c}0.47 \\
(0.35,0.62)\end{array}$ & $\begin{array}{c}0.59 \\
(0.40,0.86)\end{array}$ & $\begin{array}{c}0.47 \\
(0.24,0.92)\end{array}$ & $\begin{array}{c}1.60 \\
(1.20,2.13)\end{array}$ & $\begin{array}{c}1.09 \\
(0.76,1.58)\end{array}$ \\
\hline $\begin{array}{c}0.54 \\
(0.13,2.28)\end{array}$ & $\begin{array}{c}0.14 \\
(0.04,0.54)\end{array}$ & $\begin{array}{c}\text { Ipilimumab } \\
10 \mathrm{mg} / \mathrm{kg}\end{array}$ & $\begin{array}{c}2.70 \\
(0.89,8.23)\end{array}$ & $\begin{array}{c}3.25 \\
(1.50,7.05)\end{array}$ & $\begin{array}{c}0.68 \\
(0.33,1.40)\end{array}$ & $\begin{array}{c}0.86 \\
(0.40,1.84)\end{array}$ & $\begin{array}{c}0.69 \\
(0.27,1.76)\end{array}$ & $\begin{array}{c}2.33 \\
(1.13,4.81)\end{array}$ & $\begin{array}{c}1.60 \\
(0.79,3.24)\end{array}$ \\
\hline $\begin{array}{c}0.91 \\
(0.32,2.63)\end{array}$ & $\begin{array}{c}0.23 \\
(0.07,0.74)\end{array}$ & $\begin{array}{c}1.68 \\
(0.28,9.93)\end{array}$ & $\begin{array}{c}\text { Tremelimumab } \\
10 \mathrm{mg} / \mathrm{kg}\end{array}$ & $\begin{array}{c}1.20 \\
(0.54,2.68)\end{array}$ & $\begin{array}{c}0.25 \\
(0.10,0.64)\end{array}$ & $\begin{array}{c}0.32 \\
(0.12,0.84)\end{array}$ & $\begin{array}{c}0.25 \\
(0.08,0.77)\end{array}$ & $\begin{array}{c}0.87 \\
(0.34,2.22)\end{array}$ & $\begin{array}{c}0.59 \\
(0.23,1.53)\end{array}$ \\
\hline $\begin{array}{c}0.85 \\
(0.49,1.47)\end{array}$ & $\begin{array}{c}0.22 \\
(0.10,0.45)\end{array}$ & $\begin{array}{c}1.56 \\
(0.34,7.25)\end{array}$ & $\begin{array}{c}0.93 \\
(0.38,2.30)\end{array}$ & $\begin{array}{c}\text { Tremelimumab } \\
15 \mathrm{mg} / \mathrm{kg}\end{array}$ & $\begin{array}{c}0.21 \\
(0.13,0.33)\end{array}$ & $\begin{array}{c}0.26 \\
(0.15,0.45)\end{array}$ & $\begin{array}{c}0.21 \\
(0.10,0.45)\end{array}$ & $\begin{array}{c}0.72 \\
(0.44,1.18)\end{array}$ & $\begin{array}{c}0.49 \\
(0.30,0.81)\end{array}$ \\
\hline $\begin{array}{c}3.52 \\
(1.76,7.03)\end{array}$ & $\begin{array}{c}0.90 \\
(0.53,1.51)\end{array}$ & $\begin{array}{c}6.49 \\
(1.52,27.65)\end{array}$ & $\begin{array}{c}3.87 \\
(1.11,13.49)\end{array}$ & $\begin{array}{c}4.16 \\
(1.72,10.08)\end{array}$ & $\begin{array}{c}\text { Nivolumab } \\
3 \mathrm{mg} / \mathrm{kg}\end{array}$ & $\begin{array}{c}1.26 \\
(0.79,2.00)\end{array}$ & $\begin{array}{c}1.01 \\
(0.49,2.06)\end{array}$ & $\begin{array}{c}3.43 \\
(2.47,4.75)\end{array}$ & $\begin{array}{c}2.35 \\
(1.51,3.65)\end{array}$ \\
\hline $\begin{array}{c}2.38 \\
(0.91,6.23)\end{array}$ & $\begin{array}{c}0.61 \\
(0.21,1.78)\end{array}$ & $\begin{array}{c}4.39 \\
(0.78,24.68)\end{array}$ & $\begin{array}{c}2.62 \\
(0.63,10.94)\end{array}$ & $\begin{array}{c}2.82 \\
(0.93,8.53)\end{array}$ & $\begin{array}{c}0.68 \\
(0.21,2.21)\end{array}$ & $\begin{array}{c}\text { Pembrolizuma } \\
10 \mathrm{mg} / \mathrm{kg}\end{array}$ & $\begin{array}{c}0.80 \\
(0.42,1.52)\end{array}$ & $\begin{array}{c}2.72 \\
(1.70,4.37)\end{array}$ & $\begin{array}{c}1.87 \\
(1.12,3.12)\end{array}$ \\
\hline $\begin{array}{c}2.80 \\
(1.09,7.21)\end{array}$ & $\begin{array}{c}0.71 \\
(0.25,2.07)\end{array}$ & $\begin{array}{c}5.15 \\
(0.92,28.80)\end{array}$ & $\begin{array}{c}3.07 \\
(0.74,12.73)\end{array}$ & $\begin{array}{c}3.31 \\
(1.11,9.89)\end{array}$ & $\begin{array}{c}0.79 \\
(0.25,2.57)\end{array}$ & $\begin{array}{c}1.17 \\
(0.54,2.54)\end{array}$ & $\begin{array}{c}\text { Pembrolizuma } \\
2 \mathrm{mg} / \mathrm{kg}\end{array}$ & $\begin{array}{c}3.40 \\
(1.65,7.03)\end{array}$ & $\begin{array}{c}2.33 \\
(1.11,4.91)\end{array}$ \\
\hline $\begin{array}{c}6.71 \\
(3.27,13.75)\end{array}$ & $\begin{array}{c}1.71 \\
(1.04,2.83)\end{array}$ & $\begin{array}{c}12.36(2.92 \\
52.30)\end{array}$ & $\begin{array}{c}7.36 \\
(2.08,26.07)\end{array}$ & $\begin{array}{c}7.93 \\
(3.21,19.60)\end{array}$ & $\begin{array}{c}1.90 \\
(1.13,3.21)\end{array}$ & $\begin{array}{c}2.81 \\
(0.85,9.32)\end{array}$ & $\begin{array}{c}2.40 \\
(0.73,7.89)\end{array}$ & $\begin{array}{c}\text { Ipilimumab+ } \\
\text { Nivolumab }\end{array}$ & $\begin{array}{c}0.69 \\
(0.43,1.08)\end{array}$ \\
\hline $\begin{array}{c}3.94 \\
(2.24,6.91)\end{array}$ & $\begin{array}{c}1.01 \\
(0.56,1.80)\end{array}$ & $\begin{array}{c}7.25 \\
(1.66,31.66)\end{array}$ & $\begin{array}{c}4.32 \\
(1.30,14.32)\end{array}$ & $\begin{array}{c}4.66 \\
(2.12,10.23)\end{array}$ & $\begin{array}{c}1.12 \\
(0.51,2.46)\end{array}$ & $\begin{array}{c}1.65 \\
(0.54,5.03)\end{array}$ & $\begin{array}{c}1.41 \\
(0.47,4.24)\end{array}$ & $\begin{array}{c}0.59 \\
(0.27,1.30)\end{array}$ & $\begin{array}{l}\text { Ipilimumab + } \\
\text { Chemotherapy }\end{array}$ \\
\hline
\end{tabular}

Note: Odds ratios in the blue zone complete is for diarrhea and in the white zone for rash. The column treatment is compared with the row treatment in blue squares while it is opposite in the while squares. Numbers in parentheses indicate $95 \%$ credible intervals.

the lowest in regard to CR, PR and ORR. Besides, it also ranked the second lowest in progressive disease, only prior to ipilimumab+chemotherapy. Tremelimumab $15 \mathrm{mg} / \mathrm{kg}$ also ranked low in CR, PR and ORR, but it had a relatively high rank in progressive disease. Although ipilimumab+nivolumab had the highest response rate, patients using ipilimumab+nivolumab were not easily to show stable disease qualities. 
Patients under pembrolizumab $2 \mathrm{mg} / \mathrm{kg}$ treatment showed the lowest probability of suffering adverse effects, especially fatigue, diarrhea and nausea. The combination of Ipilimumab and nivolumab was found to be most dangerous as it had a high rank in all five of the adverse effects, they amplified the effects of pruritus, diarrhea and nausea compared to single use of nivolumab. Application of Ipilimumab $10 \mathrm{mg} / \mathrm{kg}$ was closely related to trigger

\section{Treatment Effect AAE}

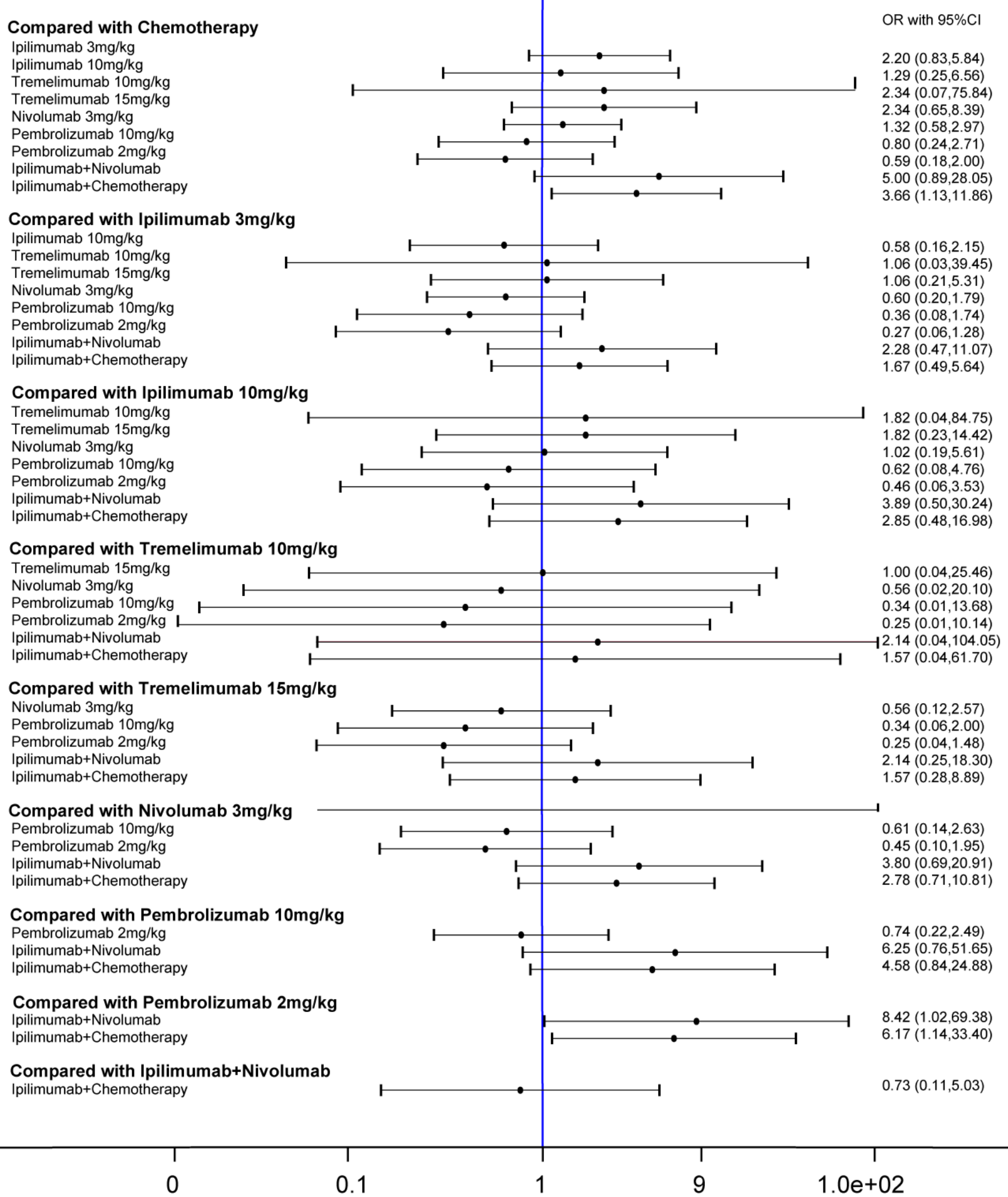

Figure 5: Forest plots for the correlations of the all-adverse-event outcome of 10 interventions on melanoma. 


\section{Treatment Effect Fatigue}

\section{Compared with Chemotherapy}

Ipilimumab $3 \mathrm{mg} / \mathrm{kg}$

Ipilimumab $10 \mathrm{mg} / \mathrm{kg}$

Tremelimumab $10 \mathrm{mg} / \mathrm{kg}$

Tremelimumab $15 \mathrm{mg} / \mathrm{kg}$

Nivolumab $3 \mathrm{mg} / \mathrm{kg}$

Pembrolizumab $10 \mathrm{mg} / \mathrm{kg}$

Pembrolizumab 2mg/kg

Ipilimumab+Nivolumab

Ipilimumab+Chemotherapy

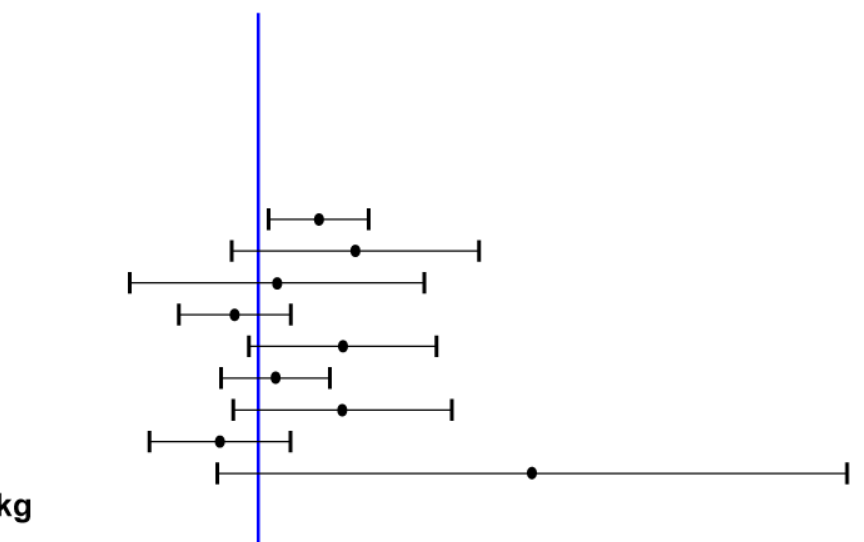

OR with $95 \% \mathrm{Cl}$

Compared with Ipilimumab $3 \mathrm{mg} / \mathbf{k g}$

Ipilimumab $10 \mathrm{mg} / \mathrm{kg}$

Tremelimumab $10 \mathrm{mg} / \mathrm{kg}$

Tremelimumab $15 \mathrm{mg} / \mathrm{kg}$

Nivolumab $3 \mathrm{mg} / \mathrm{kg}$

Pembrolizumab $10 \mathrm{mg} / \mathrm{kg}$

Pembrolizumab $2 \mathrm{mg} / \mathrm{kg}$

Ipilimumab+Nivolumab

Ipilimumab+Chemotherapy

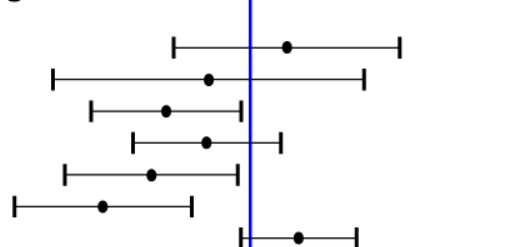

$1.54(1.08,2.20)$

$2.00(0.83,4.82)$

$1.14(0.40,3.27)$

$0.85(0.57,1.26)$

$1.83(0.94,3.57)$

$1.13(0.77,1.67)$

$1.82(0.84,3.98)$

$0.76(0.46,1.26)$

$7.05(0.75,66.50)$

Compared with Ipilimumab $10 \mathrm{mg} / \mathrm{kg}$

Tremelimumab $10 \mathrm{mg} / \mathrm{kg}$

Tremelimumab $15 \mathrm{mg} / \mathrm{kg}$

Nivolumab $3 \mathrm{mg} / \mathrm{kg}$

Pembrolizumab $10 \mathrm{mg} / \mathrm{kg}$

Pembrolizumab $2 \mathrm{mg} / \mathrm{kg}$

Ipilimumab+Nivolumab

Ipilimumab+Chemotherapy

\section{Compared with Tremelimumab $10 \mathrm{mg} / \mathrm{kg}$}

Tremelimumab $15 \mathrm{mg} / \mathrm{kg}$

Nivolumab $3 \mathrm{mg} / \mathrm{kg}$

Pembrolizumab $10 \mathrm{mg} / \mathrm{kg}$

Pembrolizumab $2 \mathrm{mg} / \mathrm{kg}$

Ipilimumab+Nivolumab

Ipilimumab+Chemotherapy

\section{Compared with Tremelimumab 15mg/kg}

Nivolumab $3 \mathrm{mg} / \mathrm{kg}$

Pembrolizumab $10 \mathrm{mg} / \mathrm{kg}$

Pembrolizumab $2 \mathrm{mg} / \mathrm{kg}$

Ipilimumab+Nivolumab

Ipilimumab+Chemotherapy

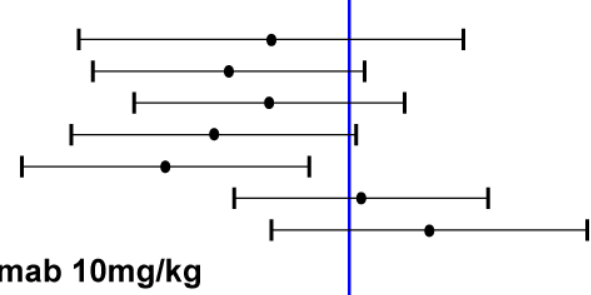

$1.30(0.58,2.91)$

$0.74(0.25,2.25)$

$0.55(0.32,0.94)$

$0.73(0.43,1.24)$

$0.49(0.27,0.92)$

$0.35(0.19,0.66)$

$1.41(0.94,2.13)$

$2.30(1.05,5.04)$

$0.57(0.15,2.25)$

$0.42(0.16,1.11)$

$0.57(0.22,1.49)$

$0.38(0.14,1.05)$

$0.27(0.10,0.75)$

$1.09(0.44,2.69)$

$1.77(0.57,5.45)$

$0.74(0.28,1.95)$

$0.99(0.32,3.03)$

$0.67(0.21,2.13)$

$0.47(0.15,1.52)$

$1.90(0.58,6.21)$

$3.09(0.79,12.03)$

$1.34(0.77,2.33)$

$0.90(0.47,1.71)$

$0.64(0.33,1.23)$

$2.57(1.31,5.05)$

$4.18(1.61,10.82)$

\section{Compared with Nivolumab $3 \mathrm{mg} / \mathrm{kg}$}

Pembrolizumab $10 \mathrm{mg} / \mathrm{kg}$

Pembrolizumab $2 \mathrm{mg} / \mathrm{kg}$

Ipilimumab+Nivolumab

Ipilimumab+Chemotherapy

Compared with Pembrolizumab 10mg/kg

Pembrolizumab $2 \mathrm{mg} / \mathrm{kg}$

Ipilimumab+Nivolumab

Ipilimumab+Chemotherapy

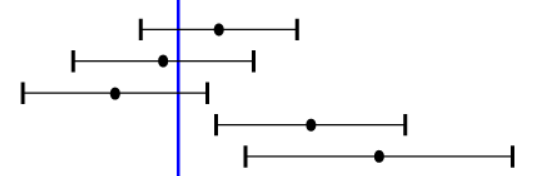

$0.67(0.36,1.27)$

$0.48(0.25,0.91)$

$1.92(0.99,3.75)$

$3.13(1.21,8.07)$

$0.71(0.42,1.21)$

$2.86(1.36,6.00)$

$4.65(1.71,12.62)$

Compared with Pembrolizumab 2mg/kg

Ipilimumab+Nivolumab

Ipilimumab+Chemotherapy
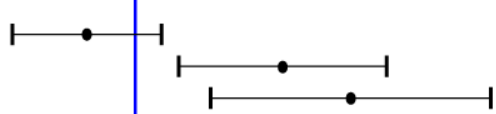

$4.03(1.90,8.58)$

$6.56(2.39,17.98)$

Compared with Ipilimumab+Nivolumab

Ipilimumab+Chemotherapy

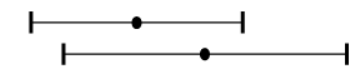

$1.63(0.67,3.95)$

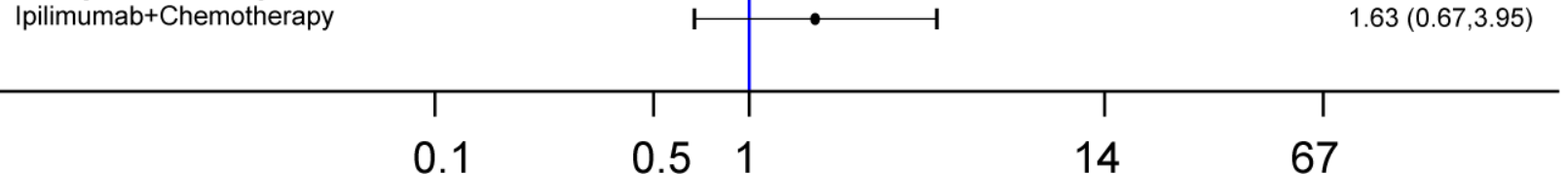

Figure 6: Forest plots for the correlations of the fatigue outcome of 10 interventions on melanoma. 
Table 7: NMA results of SUCRA for all intervention outcomes of melanoma

\begin{tabular}{lccccccccccc}
\hline Treatment & CR & PR & SD & PD & ORR & AAE & Fatigue & Pruritus & Rash & Diarrhea & Nausea \\
\hline Chemotherapy & 12.7 & 12.7 & 53.8 & 27.6 & 21.4 & 71.2 & 62.4 & 93.2 & 76.2 & 88.3 & 27.6 \\
Ipilimumab $3 \mathrm{mg} / \mathrm{kg}$ & 25.1 & 35.8 & 76.9 & 31.3 & 38.9 & 36.7 & 34.8 & 26.2 & 25.7 & 41.5 & 35.1 \\
Ipilimumab 10 mg/kg & 43.5 & 35.5 & 55.0 & 39.8 & 51.0 & 60.0 & 24.1 & 93.2 & 90.6 & 61.8 & 44.1 \\
Tremelimumab 10 mg/kg & 33.8 & 26.8 & 49.4 & 49.1 & 36.3 & 42.5 & 52.8 & 35.5 & 78.5 & 15.0 & 48.7 \\
Tremelimumab $15 \mathrm{mg} / \mathrm{kg}$ & 25.4 & 18.3 & 52.3 & 50.9 & 31.0 & 35.8 & 75.3 & 22.0 & 83.6 & 4.6 & 55.4 \\
Nivolumab 3 mg/kg & 75.8 & 68.8 & 22.9 & 56.0 & 77.1 & 57.9 & 53.1 & 58.9 & 31.9 & 84.1 & 72.0 \\
Pembrolizumab 10 mg/kg & 80.1 & 90.4 & 46.1 & 61.9 & 49.2 & 75.2 & 79.8 & 49.8 & 46.6 & 68.3 & 82.0 \\
Pembrolizumab 2 mg/kg & 71.2 & 81.7 & 50.9 & 70.3 & 50.4 & 85.5 & 96.5 & 47.1 & 38.9 & 82.9 & 95.6 \\
Ipilimumab + Nivolumab & 88.9 & 89.5 & 23.2 & 86.7 & 89.0 & 15.0 & 16.8 & 18.6 & 2.7 & 17.7 & 26.4 \\
Ipilimumab + Chemotherapy & 43.5 & 40.6 & 69.5 & 26.5 & 55.7 & 20.2 & 4.4 & 55.5 & 25.2 & 36.0 & 13.2 \\
\hline
\end{tabular}

Outcomes: CR complete response; PR partial response; SD stable disease; PD progressive disease; ORR overall response rate; AAE all adverse events.

fatigue, and chemotherapy was closely related to trigger nausea. Therefore, combined application of ipilimumab and chemotherapy caused a high possibility of both fatigue and nausea.

Funnel plots for publication bias were illustrated in Figure 9 and Figure 10. No significant bias was observed in publication.

\section{DISCUSSION}

In the current study, we investigated the therapeutic value of immunotherapy and chemotherapy on melanoma. Interventions were categorized as chemotherapy, ipilimumab $3 \mathrm{mg} / \mathrm{kg}$, ipilimumab $10 \mathrm{mg} / \mathrm{kg}$, tremelimumab $10 \mathrm{mg} / \mathrm{kg}$, tremelimumab $15 \mathrm{mg} / \mathrm{kg}$, nivolumab $3 \mathrm{mg} /$ $\mathrm{kg}$, pembrolizumab $10 \mathrm{mg} / \mathrm{kg}$, pembrolizumab $2 \mathrm{mg} / \mathrm{kg}$, ipilimumab + nivolumab and ipilimumab + chemotherapy. The outcomes assessed included CR, PR, SD, PD, ORR and adverse effects including fatigue, pruritus, rash, diarrhea and nausea. A total of 20 RCTs and 6,442 cases were involved in the study.

Chemotherapy agents such as temozolomide, dacarbazine, high-dose IL-2, paclitaxel and carboplatin are commonly used in the treatment of melanoma. According to the results, we observed that chemotherapy had the lowest response rate and was also closely related to PD than immunotherapy. Furthermore, chemotherapy had a high rank in adverse effects, especially nausea. Considering its relation to the progressive disease category and strong adverse effects, chemotherapy may not be an ideal treatment for patients with melanoma.

Tremelimumab and ipilimumab are human monoclonal antibodies of CTLA4. CTLA4 is a member of the immunoglobulin superfamily that encodes protein transmitting inhibitory signals to $\mathrm{T}$ cells. Monoclonal antibodies targeting CTLA4 can increase T cell function and induce tumor regression [33]. In a phase I/II study, both tremelimumab and ipilimumab illustrated a high therapeutic value in melanoma treatment [34, 35]. However, in a phase III trial, severe life threatening adverse effects were observed in patients dosed with ipilimumab. This observation prevented its application in our current study [36]. Nivolumab and pembrolizumab are antibodies of PD-1. PD-1 is expressed on activated T lymphocytes, B lymphocytes and NK cells and works as an immune checkpoint inhibitor. It acts by binding to its two ligands PD-L1 and PD-L2 to induce T cell tolerance [37]. Nivolumab and pembrolizumab have good performance in early phase studies and have been approved for the use in therapy of melanoma [38, 39]. In our results, tremelimumab $15 \mathrm{mg} / \mathrm{kg}$ was observed to have a low response rate, a low progressive diseases rank and a high incidence rate of adverse effects, with the exception of fatigue and rash. Our results also indicated that the combined application of ipilimumab and nivolumab had the highest response rate among all studied therapies; however, it was considered as the most dangerous form of therapy for its high rank in all adverse effects, as well as the second lowest stable disease rank. We strongly recommend the use of pembrolizumab, a drug that is used in immunotherapy. A low dose of pembrolizumab demonstrated a high response rate and had the lowest possibility of adverse effects, particularly fatigue, diarrhea and nausea. Moreover, it ranked the second highest in the progressive disease category.

We observed that when ipilimumab and nivolumab were applied simultaneously, both the response rate and adverse effects were amplified. This result was also supported by preclinical studies [40]. It was also observed that nivolumab had better outcomes in patients that had never received treatment before; whereas ipilimumab + nivolumab were more effective on patients with PD-L1-negative malignancies [26]. Furthermore, adverse effects were also magnified in patients using both ipilimumab and chemotherapy. Increased toxicity may limit the use of combined therapy. 


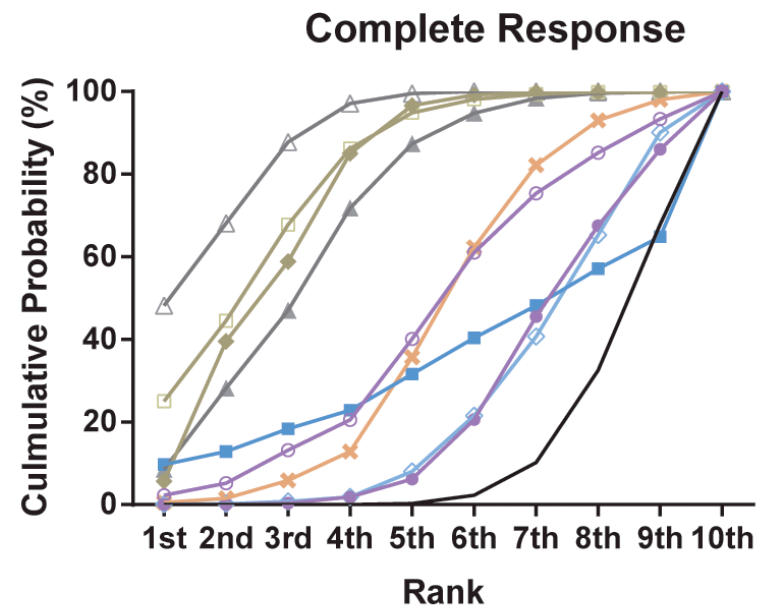

Stable Disease

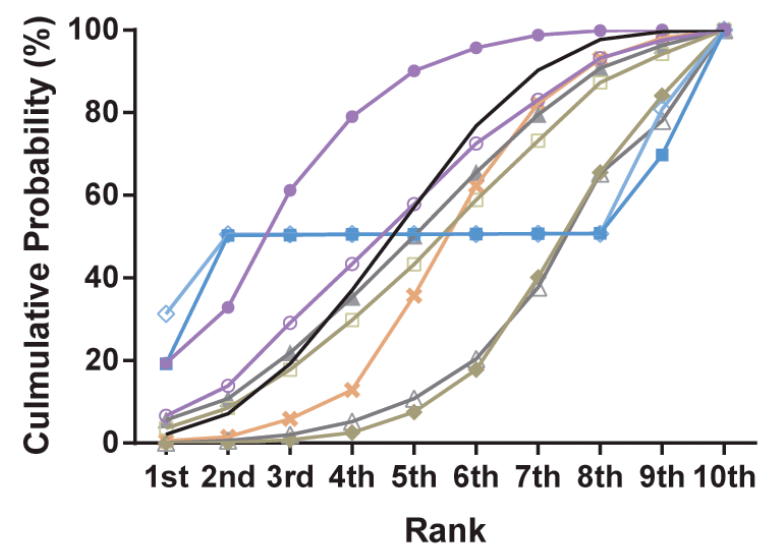

Overall Response Rate

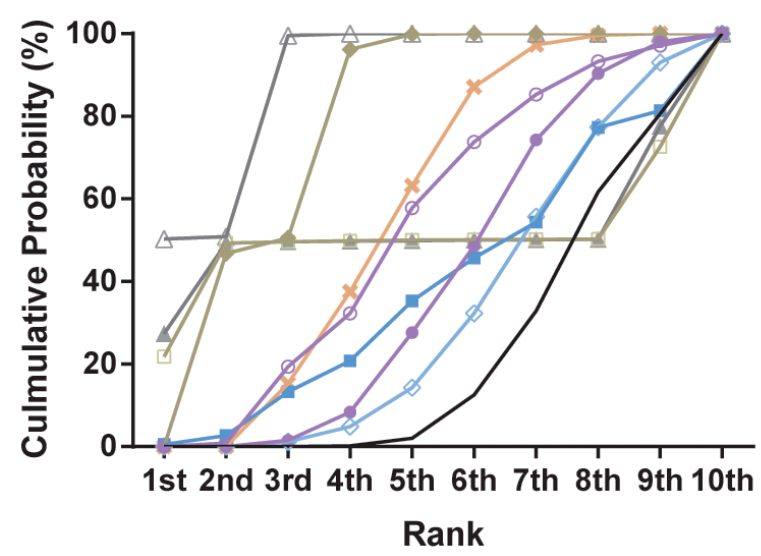

Partial Response

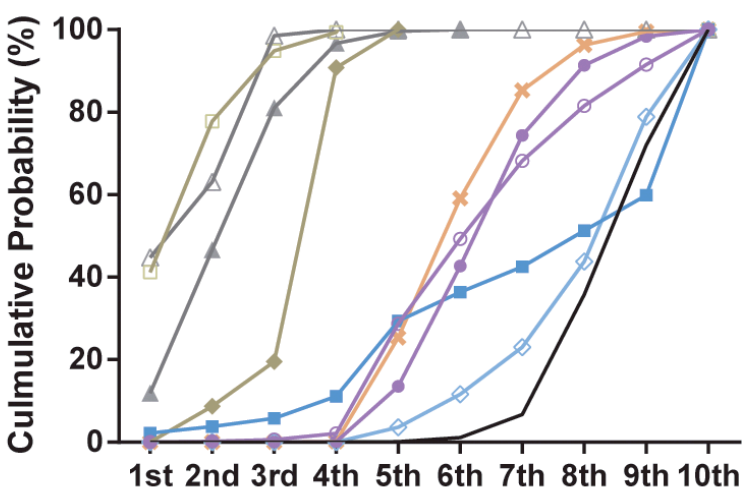

Rank

\section{Progressive Disease}

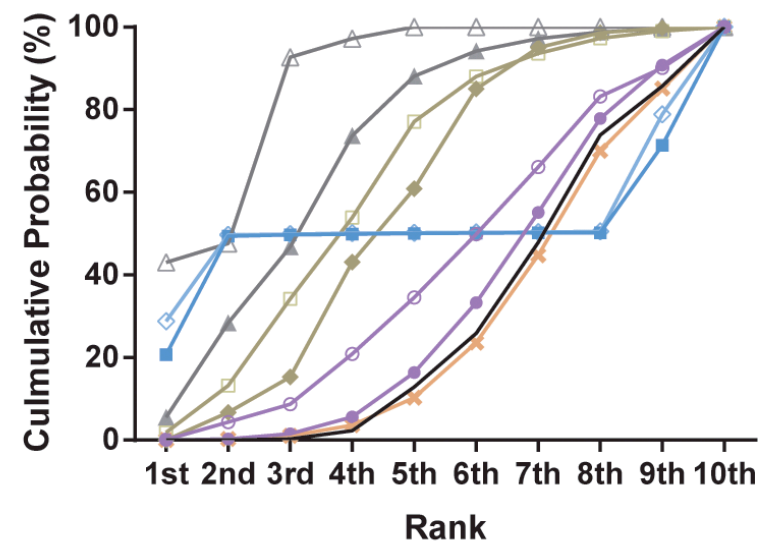

All Adverse Events

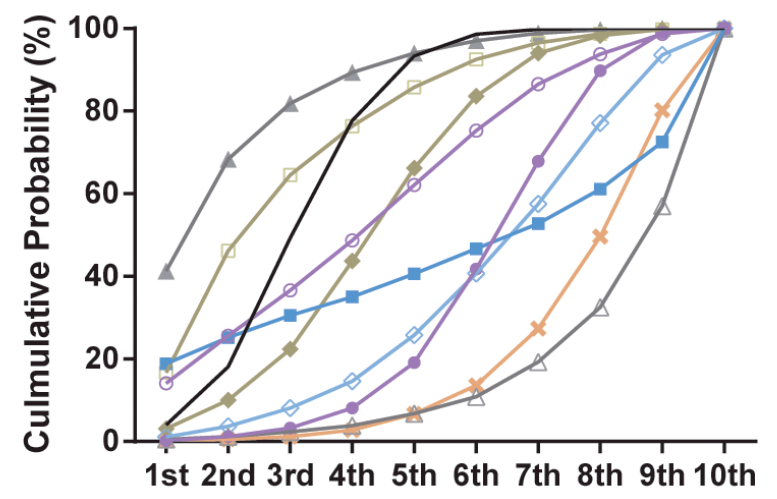

Rank

$$
\begin{aligned}
& \text { *- Ipilimumab+Chemotherapy } \\
& \neg \text { Pembrolizumab 10mg/kg } \\
& - \text { - Tremelimumab } 10 \mathrm{mg} / \mathrm{kg} \\
& \Delta \text { Ipilimumab+Nivolumab } \\
& \rightarrow \text { Nivolumab } 3 \mathrm{mg} / \mathrm{kg} \\
& ₫ \text { Pembrolizumab 2mg/kg } \\
& \diamond \text { Tremelimumab } 15 \mathrm{mg} / \mathrm{kg} \\
& \multimap \quad \text { Ipilimumab } 10 \mathrm{mg} / \mathrm{kg}
\end{aligned}
$$

Figure 7: Rankograms showing cumulative probability of each strategy having each specific rank (1-10) for clinical response. Ranking indicates the probability to be the best treatment, the second best, the third best and so on. Rank 1st is best and Rank 10 th is worst. 
There are also some limitations of our metaanalysis that should be noted. Although this is a largescale meta-analysis concerning the therapeutic value of
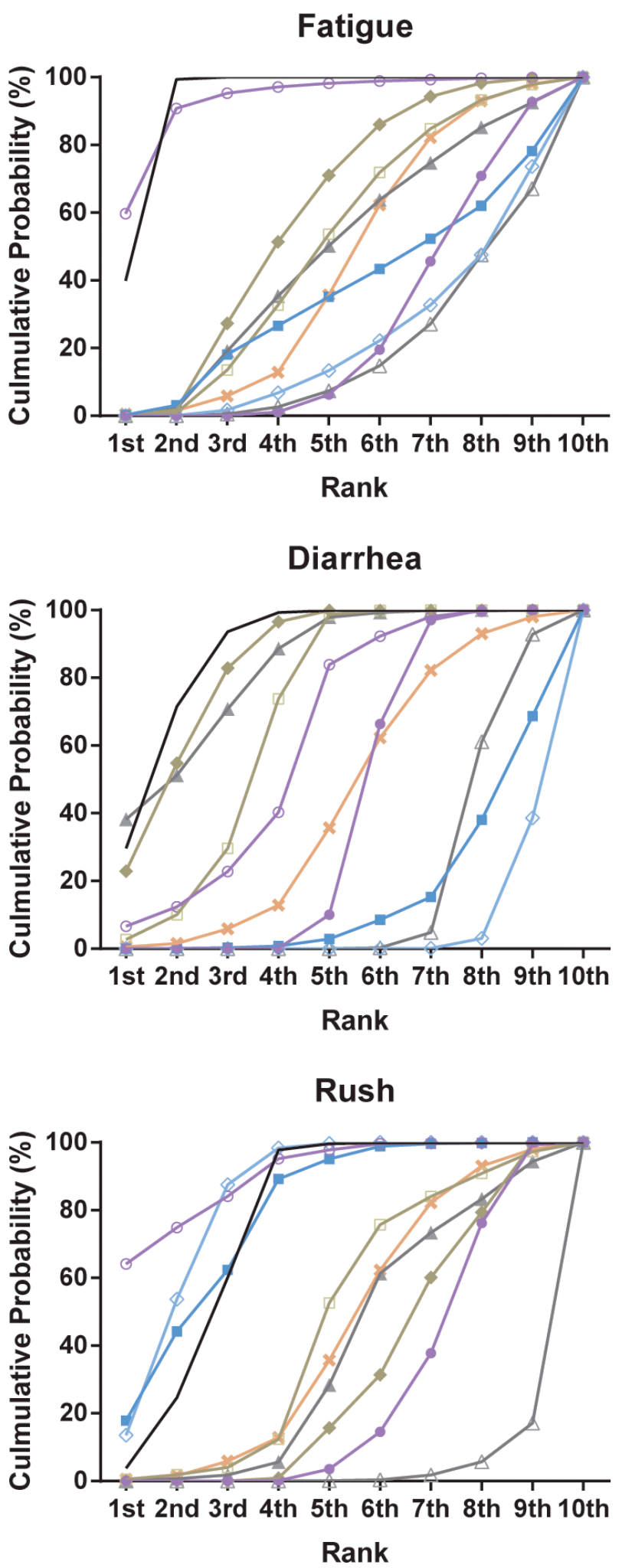

immunotherapy and chemotherapy on melanoma, the samples size is quite limited. Subgroup analysis based on the ethnicity and age of patients was not performed. The
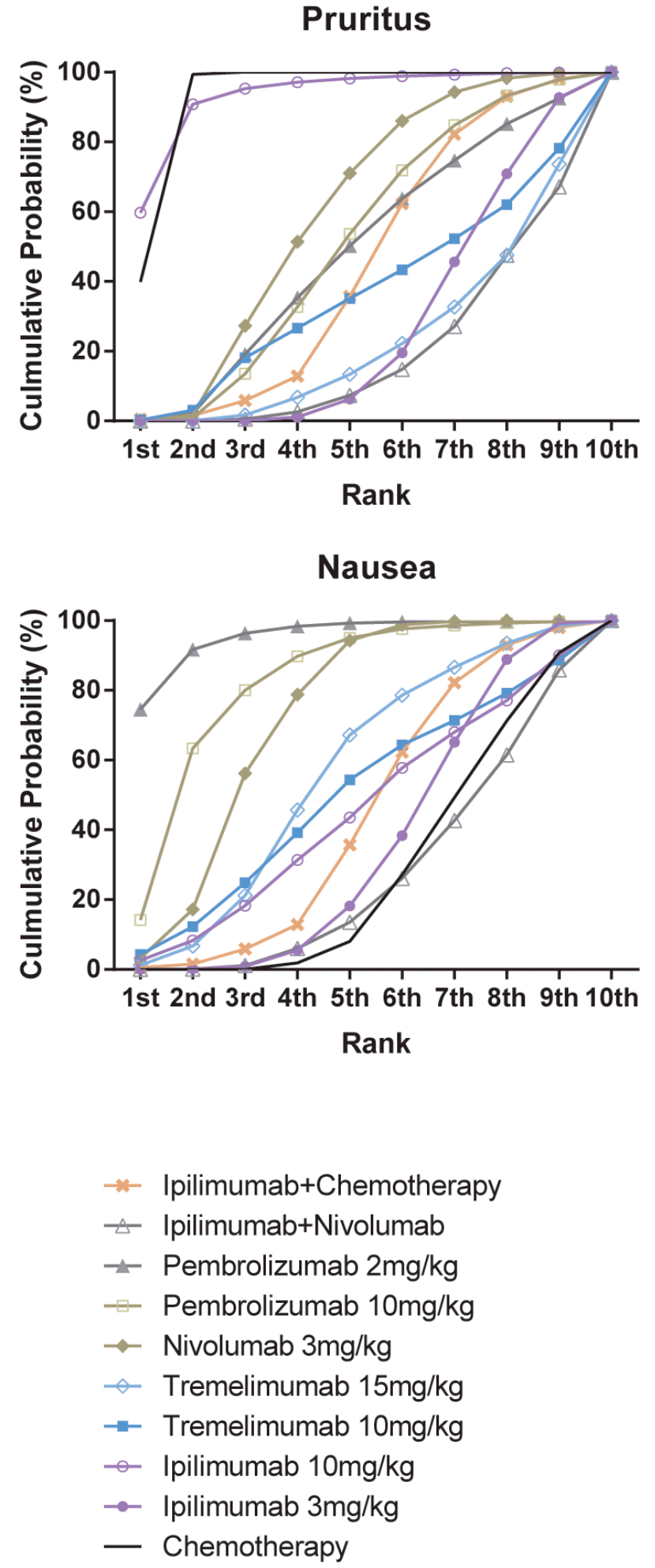

Figure 8: Rankograms showing cumulative probability of each strategy having each specific rank (1-10) for adverse events. Ranking indicates the probability to be the best treatment, the second best, the third best and so on. Rank 1st is best and Rank 10th is worst. 
lack of standardized agents in chemotherapy may also affect the reliability and validity of our results. Meanwhile, there are no sufficient data on SD and PD, which leads to several low reliable results. For example ipilimumab + nivolumab ranked low in SD but highest in PD among all. Further test of SD and PD should be determined to optimize the result. Furthermore, our study only compared the therapeutic value of CTLA4 and PD-1 inhibitors. The MEK inhibitor trametinib and BRAF inhibitors, such as vemurafenib and dabrafenib were not enrolled in the comparison as we failed to retrieve related RCTs.

In conclusion, our network meta-analysis results indicate that the combined use of immunotherapy and pembrolizumab is the treatment of choice. This is due to its high efficacy rate and minimal adverse effects. The combined application of ipilimumab and nivolumab may generate a higher incidence rate of adverse effects. Since the combined application of ipilimumab and nivolumab had the highest response rate, it should be

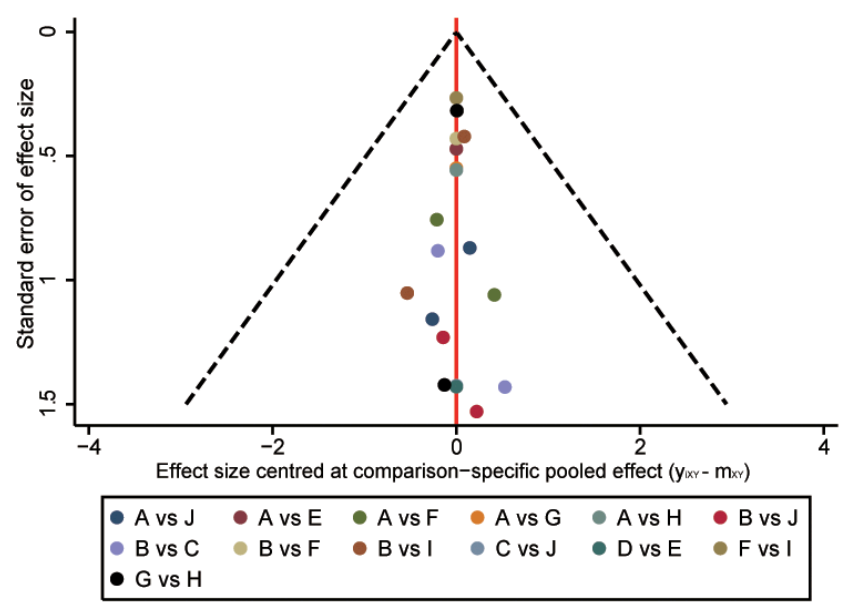

Complete Response

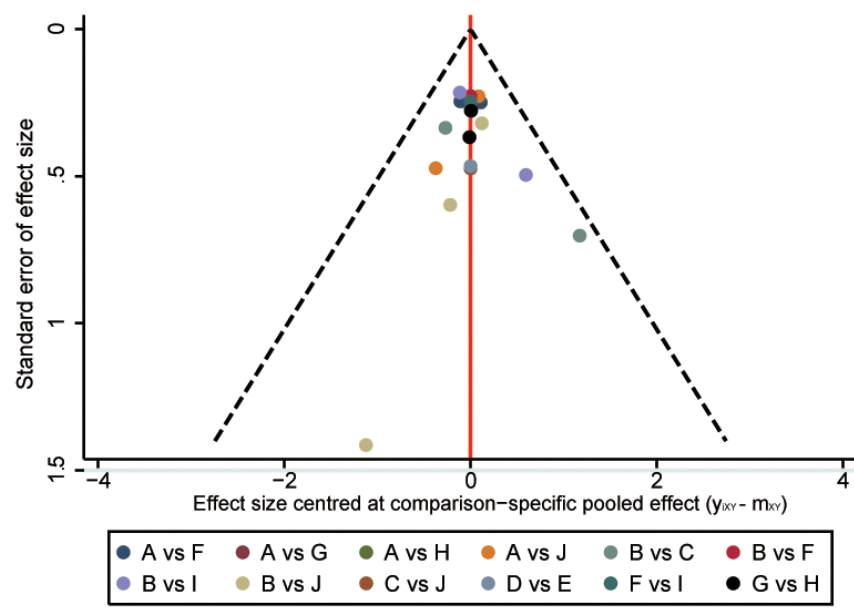

Stable Disease applied to patients that do not respond to other treatments. Chemotherapy had a low response rate, high adverse effects and progressive diseases qualities and therefore, it is not a preferred treatment for patients with melanoma.

\section{MATERIALS AND METHODS}

\section{Search strategy and inclusion criteria}

The Cochrane library, PubMed and Embase databases were used to search for any relevant articles containing the key terms: melanoma, chemotherapy, immunotherapy, ipilimumab, tremelimumab, nivolumab, pidilizumab, pembrolizumab, and randomized controlled trial. Retrieved articles were predominately screened by two independent researchers (Dr. Xinhua Wang and Dr. Ziwen Long) based on titles and abstracts. We also manually reviewed the reference list for related studies to avoid improper exclusion.

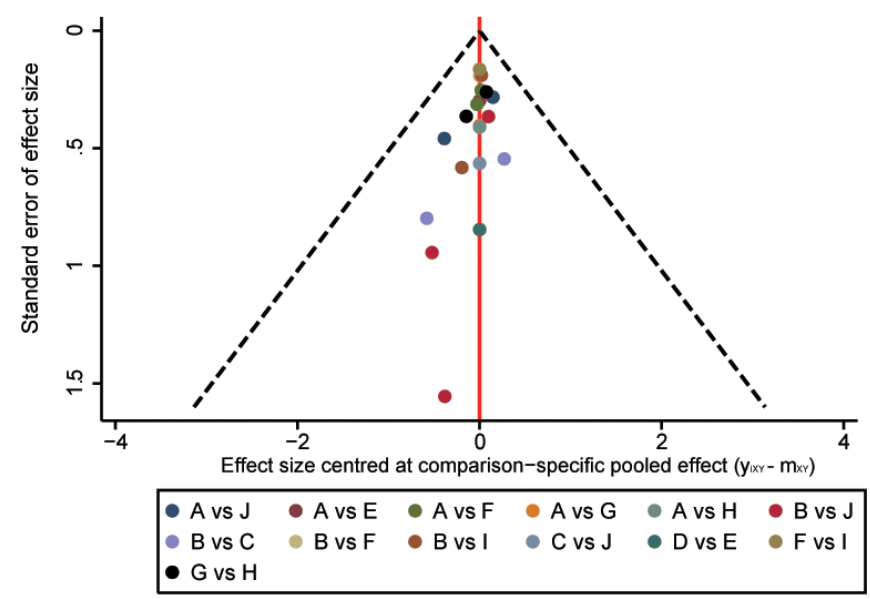

Partial Response

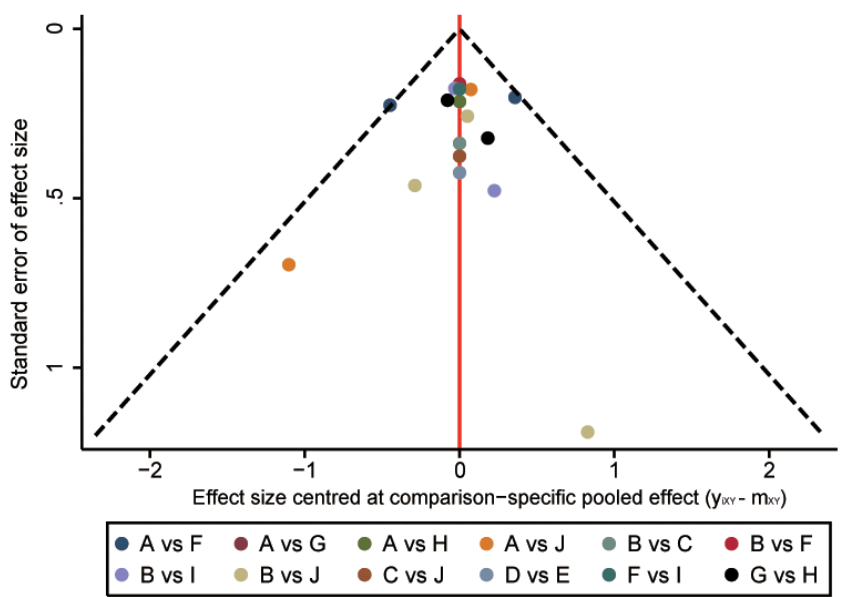

Progressive Disease

Figure 9: Funnel plot for assessing publications bias of clinical response. A Chemotherapy; B Ipilimumab $3 \mathrm{mg} / \mathrm{kg} ; \mathrm{C}$ Ipilimumab 10 mg/kg; D Tremelimumab 10 mg/kg; E Tremelimumab 15 mg/kg; F Nivolumab 3 mg/kg; G Pembrolizumab 10 mg/kg; H Pembrolizumab 2 mg/kg; I Ipilimumab+Nivolumab; J Ipilimumab+Chemotherapy. 
Articles were deemed relevant to the current study if they met the following criteria: I) experiments were randomized controlled trails (RCTs); II) all cases were
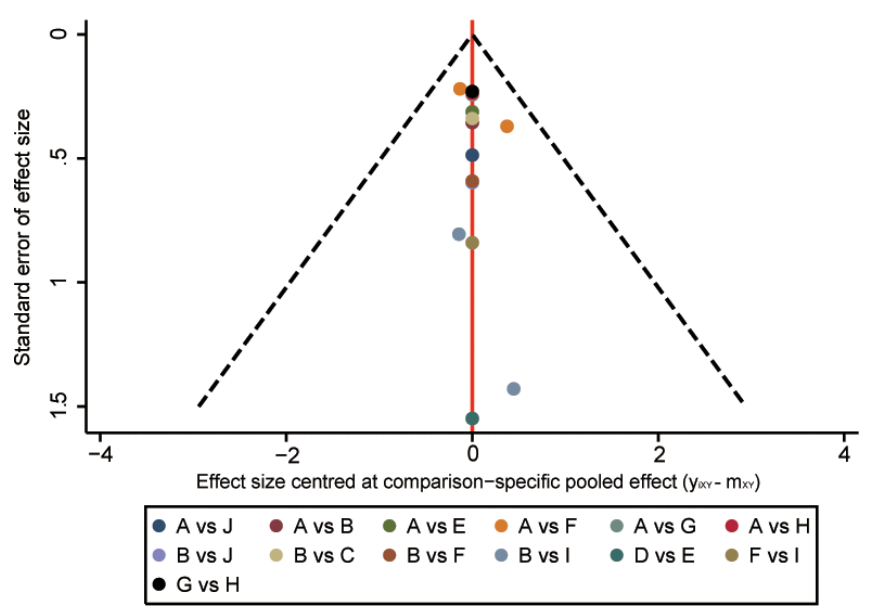

AAE

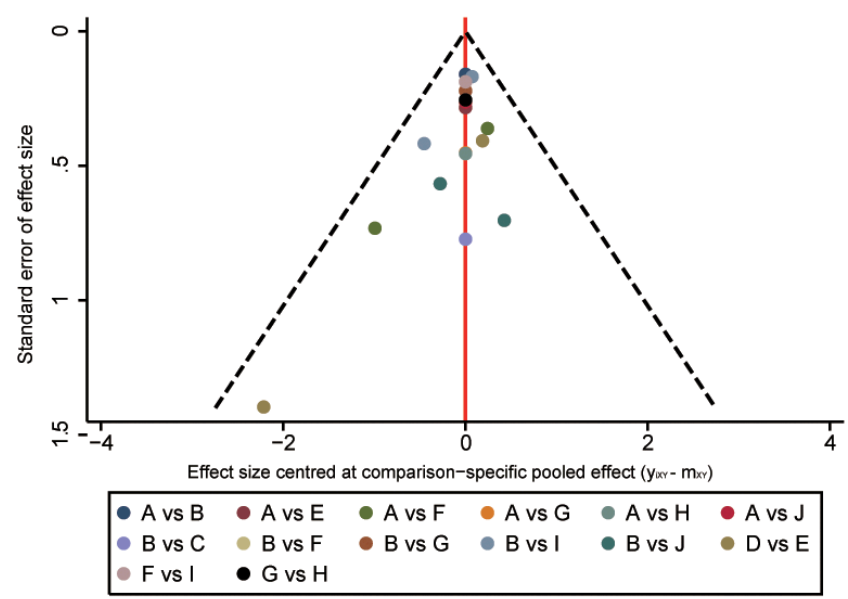

Pruritus

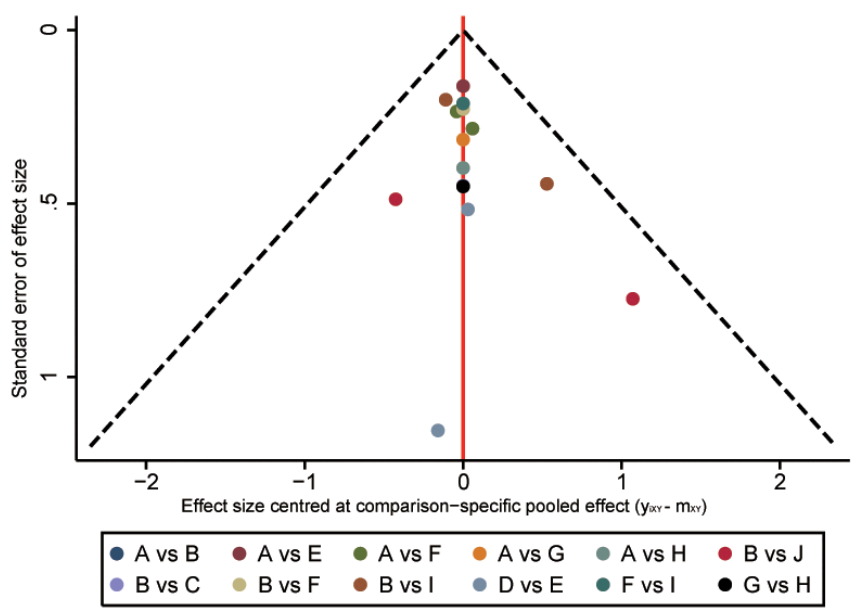

Nausea above the age of 18; III) treatment including medication and dosage was clearly described; IV) the diagnosis of melanoma is pathologically confirmed and staged

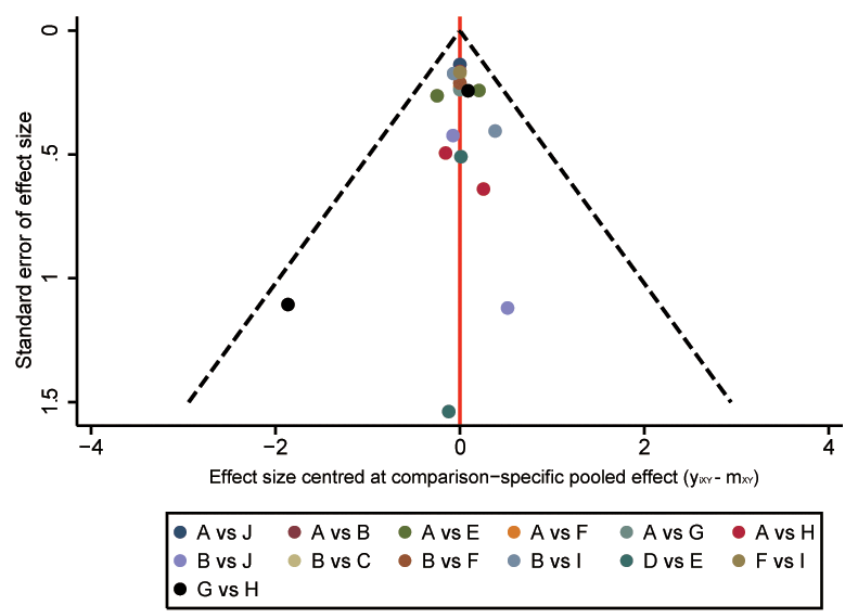

Fatigue

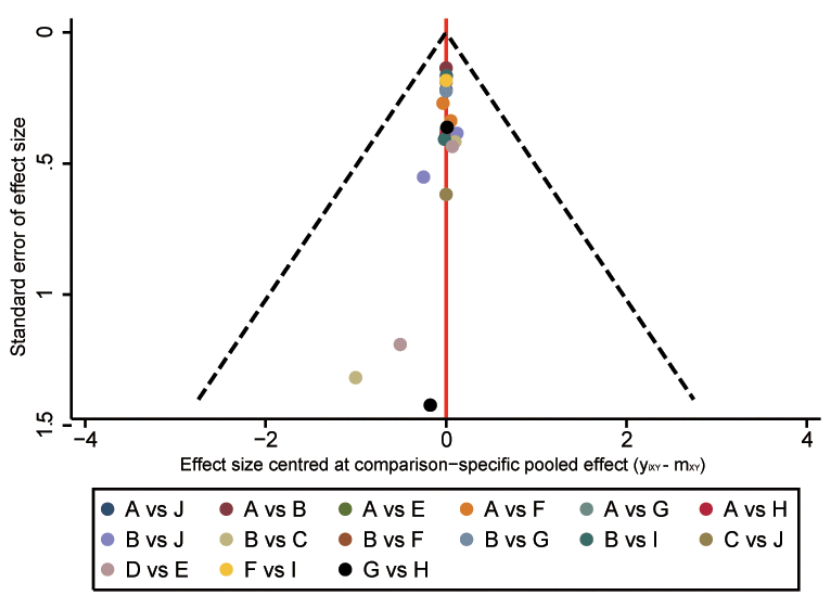

Diarrhea

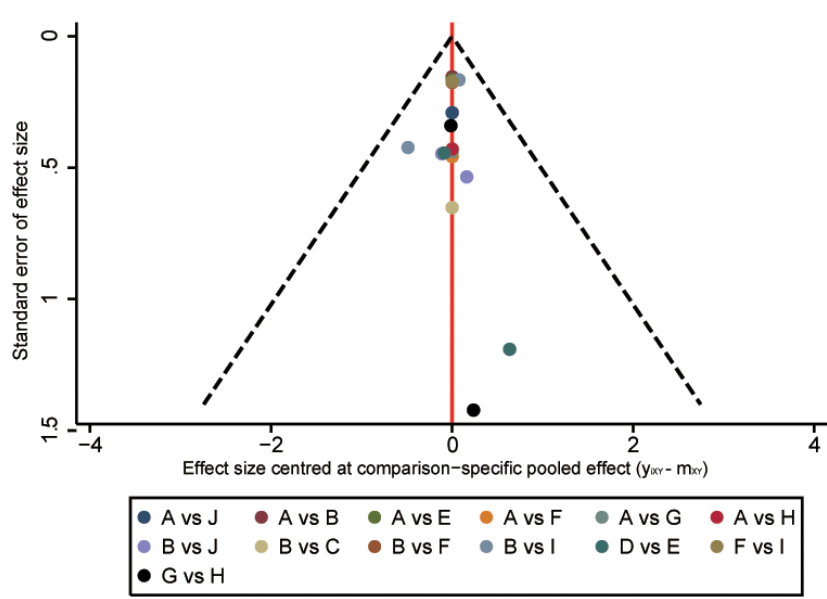

Rush

Figure 10: Funnel plot for assessing publications bias of adverse events. A Chemotherapy; B Ipilimumab $3 \mathrm{mg} / \mathrm{kg}$; C Ipilimumab 10 mg/kg; D Tremelimumab 10 mg/kg; E Tremelimumab 15 mg/kg; F Nivolumab 3 mg/kg; G Pembrolizumab 10 mg/kg; H Pembrolizumab $2 \mathrm{mg} / \mathrm{kg}$; I Ipilimumab+Nivolumab; J Ipilimumab+Chemotherapy. 
according to AJCC guidelines [9]; V) outcomes including complete response (CR), partial response (PR), stable disease $(\mathrm{SD})$, progressive disease $(\mathrm{PD})$, overall response rate (ORR) and all adverse effects (AAE) were evaluated.

The quality of all enrolled studies was assessed for bias risk using the Cochrane Collaboration's tool [41]. Studies were evaluated on their design and completeness, which included sequence generation, selective reporting, incomplete outcome data, allocation concealment, blinding and other sources of bias. Only studies with low bias risk were used in our study.

\section{Data extraction}

Two researchers (Dr. Xinhua Wang and Dr. Ziwen Long) independently extracted relevant data from the qualified articles. The data included the name of the first author, year of publication, trial ID, melanoma phase, medication used, dosage and the clinical outcome. All the data were documented for further analysis. In this study, CR, PR, SD, PD and ORR were considered as outcomes related to the effectiveness of therapy. Fatigue, pruritus, rash, diarrhea and nausea were considered as major adverse effects. A third researcher was also involved if any discrepancies arose.

\section{Statistical analysis}

Firstly, we performed a traditional pair-wise metaanalysis to evaluate therapeutic value of each treatment. Odds ratios (ORs) and corresponding 95\% confidence intervals (CIs) were calculated. The heterogeneity was determined by using Cochran's $Q$-statistic or $I^{2}$ test and a $P<0.05$ or $P^{2}>50 \%$ indicated the existence of heterogeneity. A fixed-effects model (Mantel-Haenszel method) was used for studies without significant heterogeneity, and a randomeffects model (Der Simonian-Laird method) was applied to studies with significant heterogeneity.

Subsequently, a Bayesian network meta-analysis was performed to combine both direct and indirect evidence into a single comparison, using Markov chain Monte Carlo methods. Network plots were built to illustrate the comparison of various drugs. The results were illustrated by cumulative ORs and corresponding 95\% credible intervals (CrIs). The probabilities and outcomes of each treatment were ranked and summarized using the surface under the cumulative ranking curve (SUCRA) as previously described [42].

Publication bias of involved articles was assessed using the funnel plot and Egger's test. The existence of publication bias was indicated by a $P<0.05$. In traditional pair-wise meta-analysis, we used the STATA version 12.0 (Stata Corp, College Station, TX, USA) software. And WinBUGS (MRC Bio-statistics Unit, Cambridge, UK) software was used for calculations during network meta-analysis.

\section{CONFLICTS OF INTEREST}

The authors declare no conflicts of interest.

\section{REFERENCES}

1. Saldana-Garrido JD, Martinez-Hergueta MC, MartinezRubio MM. Nevoid iris melanoma, case report. Arch Soc Esp Oftalmol. 2016.

2. Agarwal P, Nambiyar K, Manju K, Bhardwaj M. Primary Malignant Melanoma of Pleura: A Case Report and Literature Review. Diagn Cytopathol. 2016; 44:648-652.

3. Bleyer A, O'Leary M, Barr R, Ries LAG. Cancer epidemiology in older adolescents and young adults 15 to 29 years of age, including SEER incidence and survival: 1975-2000. Cancer epidemiology in older adolescents and young adults 15 to 29 years of age, including SEER incidence and survival: 1975-2000. 2006.

4. Torre LA, Bray F, Siegel RL, Ferlay J, Lortet-Tieulent J, Jemal A. Global cancer statistics, 2012. Cancer J Clin. 2015; 65:87-108.

5. Siegel RL, Miller KD, Jemal A. Cancer statistics, 2015. Cancer J Clin. 2015; 65:5-29.

6. Gandini S, Sera F, Cattaruzza MS, Pasquini P, Zanetti R, Masini C, Boyle P, Melchi CF. Meta-analysis of risk factors for cutaneous melanoma: III. Family history, actinic damage and phenotypic factors. European Journal of Cancer. 2005; 41:2040-2059.

7. Testori A, Rutkowski P, Marsden J, Bastholt L, ChiarionSileni V, Hauschild A, Eggermont AMM. Surgery and radiotherapy in the treatment of cutaneous melanoma. Annals of Oncology. 2009; 20(suppl 6):vi22-vi29.

8. Hodi FS, O'Day SJ, McDermott DF, Weber RW, Sosman JA, Haanen JB, Gonzalez R, Robert C, Schadendorf D, Hassel JC. Improved survival with ipilimumab in patients with metastatic melanoma. New England Journal of Medicine. 2010; 363:711-723.

9. Balch CM, Gershenwald JE, Soong S-j, Thompson JF, Atkins MB, Byrd DR, Buzaid AC, Cochran AJ, Coit DG, Ding S. Final version of 2009 AJCC melanoma staging and classification. Journal of clinical oncology. 2009; 27:6199-6206.

10. Morton DL, Thompson JF, Cochran AJ, Mozzillo N, Elashoff R, Essner R, Nieweg OE, Roses DF, Hoekstra HJ, Karakousis CP. Sentinel-node biopsy or nodal observation in melanoma. New England Journal of Medicine. 2006; 355:1307-1317.

11. Prieto PA, Yang JC, Sherry RM, Hughes MS, Kammula US, White DE, Levy CL, Rosenberg SA, Phan GQ. CTLA4 blockade with ipilimumab: long-term follow-up of 177 patients with metastatic melanoma. Clinical Cancer Research. 2012; 18:2039-2047.

12. Middleton MR, Grob JJ, Aaronson N, Fierlbeck G, Tilgen W, Seiter S, Gore M, Aamdal S, Cebon J, Coates A. Randomized phase III study of temozolomide versus 
dacarbazine in the treatment of patients with advanced metastatic malignant melanoma. Journal of Clinical Oncology. 2000; 18:158-158.

13. Ribas A, Camacho LH, Lopez-Berestein G, Pavlov D, Bulanhagui CA, Millham R, Comin-Anduix B, Reuben JM, Seja E, Parker CA, Sharma A, Glaspy JA, Gomez-Navarro J. Antitumor activity in melanoma and anti-self responses in a phase I trial with the anti-cytotoxic T lymphocyte-associated antigen 4 monoclonal antibody CP-675,206. J Clin Oncol. 2005; 23:8968-8977.

14. Camacho LH, Antonia S, Sosman J, Kirkwood JM, Gajewski TF, Redman B, Pavlov D, Bulanhagui C, Bozon VA, Gomez-Navarro J, Ribas A. Phase I/II trial of tremelimumab in patients with metastatic melanoma. J Clin Oncol. 2009; 27:1075-1081.

15. Weber J, Thompson JA, Hamid O, Minor D, Amin A, Ron I, Ridolfi R, Assi H, Maraveyas A, Berman D, Siegel J, O'Day SJ. A randomized, double-blind, placebo-controlled, phase II study comparing the tolerability and efficacy of ipilimumab administered with or without prophylactic budesonide in patients with unresectable stage III or IV melanoma. Clin Cancer Res. 2009; 15:5591-5598.

16. Wolchok JD, Neyns B, Linette G, Negrier S, Lutzky J, Thomas L, Waterfield W, Schadendorf D, Smylie M, Guthrie T Jr., Grob JJ, Chesney J, et al. Ipilimumab monotherapy in patients with pretreated advanced melanoma: a randomised, double-blind, multicentre, phase 2, doseranging study. Lancet Oncol. 2010; 11:155-164.

17. Hamid O, Schmidt H, Nissan A, Ridolfi L, Aamdal S, Hansson J, Guida M, Hyams DM, Gomez H, Bastholt L, Chasalow SD, Berman D. A prospective phase II trial exploring the association between tumor microenvironment biomarkers and clinical activity of ipilimumab in advanced melanoma. J Transl Med. 2011; 9:204.

18. Hersh EM, O’Day SJ, Powderly J, Khan KD, Pavlick AC, Cranmer LD, Samlowski WE, Nichol GM, Yellin MJ, Weber JS. A phase II multicenter study of ipilimumab with or without dacarbazine in chemotherapy-naïve patients with advanced melanoma. Investigational New Drugs. 2011; 29:489-498.

19. Robert C, Thomas L, Bondarenko I, O'Day S, Weber J, Garbe C, Lebbe C, Baurain JF, Testori A, Grob JJ, Davidson N, Richards J, Maio M, et al. Ipilimumab plus dacarbazine for previously untreated metastatic melanoma. N Engl J Med. 2011; 364:2517-2526.

20. Millward M, Underhill C, Lobb S, McBurnie J, Meech SJ, Gomez-Navarro J, Marshall MA, Huang B, Mather CB. Phase I study of tremelimumab (CP-675 206) plus PF3512676 (CPG 7909) in patients with melanoma or advanced solid tumours. Br J Cancer. 2013; 108:1998-2004.

21. Ribas A, Kefford R, Marshall MA, Punt CJ, Haanen JB, Marmol M, Garbe C, Gogas H, Schachter J, Linette G, Lorigan P, Kendra KL, Maio M, et al. Phase III randomized clinical trial comparing tremelimumab with standard-ofcare chemotherapy in patients with advanced melanoma. J Clin Oncol. 2013; 31:616-622.
22. Hodi FS, Lee S, McDermott DF, Rao UN, Butterfield LH, Tarhini AA, Leming P, Puzanov I, Shin D, Kirkwood JM. Ipilimumab plus sargramostim vs ipilimumab alone for treatment of metastatic melanoma: a randomized clinical trial. JAMA. 2014; 312:1744-1753.

23. Robert C, Ribas A, Wolchok JD, Hodi FS, Hamid O, Kefford R, Weber JS, Joshua AM, Hwu WJ, Gangadhar TC, Patnaik A, Dronca R, Zarour H, et al. Anti-programmeddeath-receptor-1 treatment with pembrolizumab in ipilimumab-refractory advanced melanoma: a randomised dose-comparison cohort of a phase 1 trial. Lancet. 2014; 384:1109-1117.

24. Topalian SL, Sznol M, McDermott DF, Kluger HM, Carvajal RD, Sharfman WH, Brahmer JR, Lawrence DP, Atkins MB, Powderly JD, Leming PD, Lipson EJ, Puzanov I, et al. Survival, durable tumor remission, and long-term safety in patients with advanced melanoma receiving nivolumab. J Clin Oncol. 2014; 32:1020-1030.

25. Eggermont AM, Chiarion-Sileni V, Grob JJ, Dummer R, Wolchok JD, Schmidt H, Hamid O, Robert C, Ascierto PA, Richards JM, Lebbe C, Ferraresi V, Smylie M, et al. Adjuvant ipilimumab versus placebo after complete resection of high-risk stage III melanoma (EORTC 18071): a randomised, double-blind, phase 3 trial. Lancet Oncol. 2015; 16:522-530.

26. Larkin J, Chiarion-Sileni V, Gonzalez R, Grob JJ, Cowey CL, Lao CD, Schadendorf D, Dummer R, Smylie M, Rutkowski P, Ferrucci PF, Hill A, Wagstaff J, et al. Combined Nivolumab and Ipilimumab or Monotherapy in Untreated Melanoma. N Engl J Med. 2015; 373:23-34.

27. Maio M, Grob JJ, Aamdal S, Bondarenko I, Robert C, Thomas L, Garbe C, Chiarion-Sileni V, Testori A, Chen TT, Tschaika M, Wolchok JD. Five-year survival rates for treatment-naive patients with advanced melanoma who received ipilimumab plus dacarbazine in a phase III trial. J Clin Oncol. 2015; 33:1191-1196.

28. Postow MA, Chesney J, Pavlick AC, Robert C, Grossmann K, McDermott D, Linette GP, Meyer N, Giguere JK, Agarwala SS, Shaheen M, Ernstoff MS, Minor D, et al. Nivolumab and ipilimumab versus ipilimumab in untreated melanoma. N Engl J Med. 2015; 372:2006-2017.

29. Ribas A, Puzanov I, Dummer R, Schadendorf D, Hamid O, Robert C, Hodi FS, Schachter J, Pavlick AC, Lewis KD, Cranmer LD, Blank CU, O’Day SJ, et al. Pembrolizumab versus investigator-choice chemotherapy for ipilimumabrefractory melanoma (KEYNOTE-002): a randomised, controlled, phase 2 trial. Lancet Oncol. 2015; 16:908-918.

30. Robert C, Long GV, Brady B, Dutriaux C, Maio M, Mortier L, Hassel JC, Rutkowski P, McNeil C, Kalinka-Warzocha E, Savage KJ, Hernberg MM, Lebbe C, et al. Nivolumab in previously untreated melanoma without BRAF mutation. $\mathrm{N}$ Engl J Med. 2015; 372:320-330.

31. Robert C, Schachter J, Long GV, Arance A, Grob JJ, Mortier L, Daud A, Carlino MS, McNeil C, Lotem M, 
Larkin J, Lorigan P, Neyns B, et al. Pembrolizumab versus Ipilimumab in Advanced Melanoma. N Engl J Med. 2015; 372:2521-2532.

32. Weber JS, D’Angelo SP, Minor D, Hodi FS, Gutzmer R, Neyns B, Hoeller C, Khushalani NI, Miller WH Jr., Lao CD, Linette GP, Thomas L, et al. Nivolumab versus chemotherapy in patients with advanced melanoma who progressed after anti-CTLA-4 treatment (CheckMate 037): a randomised, controlled, open-label, phase 3 trial. Lancet Oncol. 2015; 16:375-384.

33. Buchbinder E, Hodi FS. Cytotoxic T lymphocyte antigen-4 and immune checkpoint blockade. J Clin Invest. 2015; 125:3377-3383.

34. Ribas A. Clinical development of the anti-CTLA-4 antibody tremelimumab. Seminars in oncology: Elsevier, pp. $450-454$.

35. Weber JS, O'Day S, Urba W, Powderly J, Nichol G, Yellin M, Snively J, Hersh E. Phase I/II study of ipilimumab for patients with metastatic melanoma. Journal of Clinical Oncology. 2008; 26:5950-5956.

36. Pierard GE, Aubin F, Humbert P. Ipilimumab, a promising immunotherapy with increased overall survival in metastatic melanoma? Dermatol Res Pract. 2012; 2012:182157.

37. Okazaki T, Chikuma S, Iwai Y, Fagarasan S, Honjo T. A rheostat for immune responses: the unique properties of PD-1 and their advantages for clinical application. Nat Immunol. 2013; 14:1212-1218.
38. Hamid O, Robert C, Daud A, Hodi FS, Hwu W-J, Kefford R, Wolchok JD, Hersey P, Joseph RW, Weber JS. Safety and tumor responses with lambrolizumab (anti-PD1) in melanoma. New England Journal of Medicine. 2013; 369:134-144.

39. Robert C, Long GV, Brady B, Dutriaux C, Maio M, Mortier L, Hassel JC, Rutkowski P, McNeil C, KalinkaWarzocha E. Nivolumab in previously untreated melanoma without BRAF mutation. New England journal of medicine. $2015 ; 372: 320-330$.

40. Wolchok JD, Kluger H, Callahan MK, Postow MA, Rizvi NA, Lesokhin AM, Segal NH, Ariyan CE, Gordon RA, Reed K, Burke MM, Caldwell A, Kronenberg SA, et al. Nivolumab plus ipilimumab in advanced melanoma. N Engl J Med. 2013; 369:122-133.

41. Higgins JPT GS, eds. Cochrane Handbook for Systematic Reviews of Interventions. Version 5.1.0. The Cochrane Collaboration, . 2011.

42. Salanti G, Ades AE, Ioannidis JP. Graphical methods and numerical summaries for presenting results from multipletreatment meta-analysis: an overview and tutorial. J Clin Epidemiol. 2011; 64:163-171. 